\title{
Assortment Rotation and the Value of Concealment
}

\section{Citation}

Ferreira, Kris Johnson, and Joel Goh. "Assortment Rotation and the Value of Concealment." Harvard Business School Working Paper, No. 17-041, November 2016.

\section{Permanent link}

http://nrs.harvard.edu/urn-3:HUL.InstRepos:30861196

\section{Terms of Use}

This article was downloaded from Harvard University's DASH repository, and is made available under the terms and conditions applicable to Open Access Policy Articles, as set forth at http:// nrs.harvard.edu/urn-3:HUL.InstRepos:dash.current.terms-of-use\#OAP

\section{Share Your Story}

The Harvard community has made this article openly available.

Please share how this access benefits you. Submit a story.

Accessibility 


$$
\text { H A R VAR D B US INESS S C HOOL }
$$

\section{Assortment Rotation and the Value of Concealment}

Kris Johnson Ferreira Joel Goh

Working Paper 17-041 


\title{
Assortment Rotation and the Value of Concealment
}

\author{
Kris Johnson Ferreira
}

Harvard Business School

Joel Goh

Harvard Business School

Working Paper 17-041 


\title{
Assortment Rotation and the Value of Concealment
}

\author{
Kris Johnson Ferreira \\ Harvard Business School, kferreira@hbs.edu \\ Joel Goh \\ Harvard Business School, jgoh@hbs.edu
}

\begin{abstract}
Assortment rotation - the retailing practice of changing the assortment of products offered to customers - has recently been used as a competitive advantage for both brick-and-mortar and online retailers. Fast-fashion retailers have differentiated themselves by rotating their assortment multiple times throughout a standard selling season. Interestingly, the entire online flash sales industry was created using this idea as a cornerstone of its business strategy. In this paper we identify and investigate a new reason why frequent assortment rotations can be valuable to a retailer, particularly for products where consumers typically purchase multiple products in a given category during a selling season. Namely, by distributing its seasonal catalog of products over multiple assortments rotated throughout the season - as opposed to selling all products in a single assortment - the retailer effectively conceals a portion of its full product catalog from consumers. This injects uncertainty into the consumer's relative product valuations since she is unable to observe the entire catalog of products that the retailer will sell that season. Rationally-acting consumers may respond to this additional uncertainty by purchasing more products, thereby generating additional sales for the retailer. We refer to this phenomenon as the value of concealment. A negative value of concealment is possible and represents the event that rationally-acting consumers respond to the additional uncertainty by purchasing fewer products. We develop a consumer choice model and finite-horizon stochastic dynamic program to study when the value of concealment is positive or negative. We show that when consumers are myopic, the value of concealment is always positive. In contrast, we show that when consumers are strategic, the value of concealment is context-dependent; we present insights and discuss intuition regarding which product categories likely lead to positive vs. negative values of concealment.
\end{abstract}

Keywords: assortment planning, strategic consumers, consumer choice

\section{Introduction}

Assortment rotation - the retailing practice of changing the assortment of products offered to customers throughout a selling season - has recently been used by both brick-and-mortar and online retailers as a strategy for gaining competitive advantage. A notable category of retailers who have employed this strategy successfully are "fast fashion retailers" such as Zara and H\&M, who have differentiated themselves from other retailers by rotating their assortment multiple times throughout the fashion industry standard 6-month selling season. As shown in Figure 1, these two 


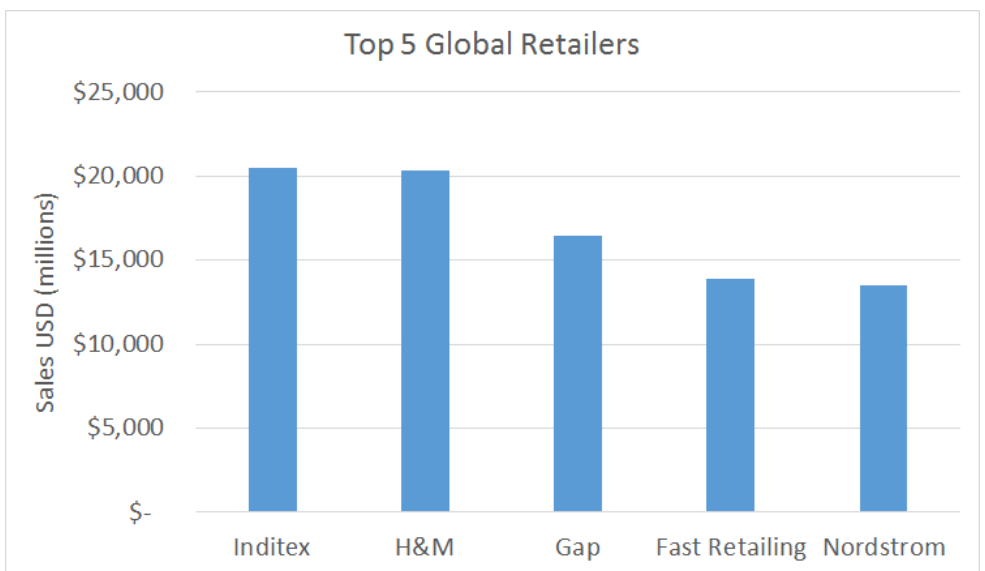

Figure 1 Largest Global Fashion Retailers by Revenue in 2015 (Chang et al. 2016).

companies were the two largest global fashion retailers in terms of revenue earned in $2015^{1}$ (Chang et al. 2016). The success of this fast fashion strategy, where assortments are frequently rotated, has led other retailers to try and replicate this strategy to remain competitive. Gap announced that it would be testing the fast fashion model in select stores in Spring 2016 (Kapner 2015), and Nordstrom has partnered with the fast fashion retailer Topshop in the United Kingdom to transform a portion of its stores to mimic the fast fashion model (Dishman 2012). Interestingly, the entire industry of online flash sales (e.g., Rue La La and Groupon) has been created using the idea of frequent assortment rotation as a cornerstone of its business strategy: It is not uncommon for such companies to rotate their assortments even on a daily basis.

Several reasons have been proposed by both practitioners and academics explaining the recent rise and success of frequent assortment rotations. We briefly outline a few of these main ways that frequent rotations can add value to retailers: First, for brick-and-mortar retailers who face limited shelf space constraints, frequent assortment rotations effectively allow them to offer a wider variety of products throughout a given selling season, thereby giving customers more options and increasing the likelihood that they purchase more products. Second, by frequently rotating assortments, retailers can also mitigate the challenge of predicting trends and product demand; if retailers have a short enough sourcing and production cycle, they are able to adapt assortments rapidly based on the latest trends and recent historical sales data (Fisher and Raman 1996, Kapner 2015). Third, retailers can potentially time new product introductions with consumer budget constraints; that way, consumers are able to spend their monthly budget on new products as opposed to products introduced in previous months that they were unable to afford at the time, assuming that product attractiveness decays over time (Caro et al. 2014, Caro and Martínez-de-Albéniz 2012).

\footnotetext{
${ }^{1}$ Inditex is the parent company of Zara.
} 
Much of the existing research on assortment rotation strategies (reviewed in detail in Section 1.1) focuses on product categories where consumers typically purchase at most one product until that product needs replacement, such as automobiles, home appliances, and many consumer packaged goods and grocery items. In this paper, we study the problem of assortment planning in a context that has received little research attention to date: the setting of product categories where consumers typically make multiple purchases over a selling season. Examples of these types of product categories include many apparel categories (such as boots, dresses, or swimwear), accessories, and children's toys. By doing so, we uncover a new reason why frequent assortment rotations can be valuable to a retailer. Namely, by distributing its seasonal catalog of products over multiple assortments rotated throughout the selling season - as opposed to selling all products in a single assortment over the entire season - the retailer effectively conceals a portion of its full product catalog from consumers. This injects uncertainty into the consumer's relative product valuations since she is unable to observe the entire catalog of products that the retailer will sell that season. Rationally-acting consumers may respond to this additional uncertainty by purchasing more products, thereby generating additional sales for the retailer. Throughout this paper, for the sake of brevity, we will refer to this as the retailer's value of concealment. A negative value of concealment is possible and represents the event that rationally-acting consumers respond to the additional uncertainty by purchasing fewer products; this would constitute a reason for a retailer to rotate its assortment less frequently, if at all.

The following example illustrates the value of concealment for a retailer. Consider a customer who would like to purchase at least one new pair of sandals for the summer. When a fast fashion or flash sales retailer starts selling sandals in March, the customer must decide which pair(s) of sandals to purchase without knowing the new styles of sandals that the retailer will offer in April. It is precisely this uncertainty that may cause the customer to purchase a pair of sandals in March, only to return to the store in April and purchase another pair of sandals that she loves even more; had she been able to see all styles of sandals when she had first visited the store in March, perhaps she only would have purchased her favorite pair. In this situation, the retailer sold two pairs of sandals instead of one, simply by offering different assortments each month.

The main objective of this research is two-fold: $(i)$ develop and analyze a model that captures the consumer's purchase decisions throughout a selling season when she considers purchasing multiple products in the same category, and (ii) gain an understanding around the drivers that influence the value of concealment for a retailer, i.e., the circumstances under which a retailer increases/decreases 
its expected sales simply by increasing the number of assortments offered throughout the season for the same catalog of products.

To do this, we analyze two extreme assortment rotation strategies that the retailer could choose:

1. Single assortment strategy: offer all products concurrently throughout the entire selling season.

2. Sequential assortment strategy: offer each product sequentially over the course of the selling season, only offering one product at a time to consumers. Under this assortment rotation strategy, we separately analyze the cases of myopic and strategic consumers.

We study these extremes partly for the sake of analytical tractability, and partly because the clear distinction between these two strategies is helpful in generating sharper managerial insights from our analysis. Nevertheless, we note that these extremes are not too unrealistic: the sequential assortment strategy is akin to the fast fashion and flash sales models where the retailer frequently rotates assortments, whereas the single assortment strategy is akin to the more traditional retail model of offering all products concurrently.

To achieve our first research objective, we borrow aspects of consumer utility theory from the economics literature. We use a random utility model to characterize the consumer's utility for purchasing a product. The deterministic component of the utility model is known to the customer and retailer a priori and captures diminishing returns from each additional product purchased. The random component of the utility model represents the heterogeneity in valuations across different products. It is unknown to both the retailer and consumer a priori and thus reflects the consumer's uncertainty about future product options; the consumer realizes this random component, and thus her valuation of the product, only after the retailer offers the product in an assortment. We characterize consumers' optimal purchasing policies for each assortment rotation strategy and show that in both cases they have threshold structures.

To achieve our second research objective, we consider a retailer who would like to sell a fixed set of products (in a certain category) during a finite-horizon selling season. The retailer must choose to follow either the single assortment strategy or the sequential assortment strategy in order to maximize its revenue over the course of the season. We define the value of concealment for the retailer as the difference in expected revenue between the sequential assortment strategy and single assortment strategy, and show that when consumers are myopic, the value of concealment is always positive (i.e., it is always advantageous for the retailer to offer products sequentially). Furthermore, we show that strategic consumers are always less profitable to the retailer than myopic consumers. Finally, we show that when consumers are strategic, the value of concealment can be either positive or negative, and therefore the revenue-maximizing assortment rotation strategy of the retailer is 
context-dependent. We present insights and discuss intuition regarding which product categories likely lead to positive vs. negative values of concealment.

Our work contributes to the literature and to practice in several dimensions. First, our work extends the scarce literature analyzing assortment rotation strategies where a consumer's purchase decisions in each period affect her purchase decisions in subsequent periods. To the best of our knowledge, our paper is the first to consider such a setting where consumers may purchase multiple products within the same category during the selling season. Second, our work expands upon the limited research on strategic consumers who are "strategic" with respect to their unknown future product valuations, as opposed to customers who are "strategic" about unknown future price or inventory availability, which are more commonly studied in the operations management literature. Third, our work identifies and studies a new reason why some retailers may want to rotate assortments throughout the selling season: to capture the value of concealment. Finally, our work provides practical insights to retailers to help guide their assortment rotation strategy decisions.

In the remainder of this section, we review the literature that is related to our work. In Section 2, we define the model, notation, and assumptions that lay the foundation for this research. In Section 3 , we present the optimal consumer's purchase decisions for each assortment rotation strategy that the retailer may employ, considering both myopic and strategic consumers. In Section 4, we explore its optimal assortment rotation strategy by evaluating its value of concealment, separately analyzing the cases when consumers are myopic and when they are strategic.

\subsection{Literature Review}

Our work is primarily related to the literature on how retailers should change their assortments over time, as well as the literature on strategic consumers. The paper that is most closely related to ours is Bernstein and Martínez-de-Albéniz (2016), where they also study the retailer's optimal assortment rotation strategy and the value of concealment in the presence of strategic consumers; the main difference is that they only allow for each consumer to purchase at most one product throughout the selling season. They study the tradeoff that a consumer faces between purchasing a product of known valuation now versus waiting to purchase a product with potentially higher valuation in the future; each time a consumer visits the retailer to realize her valuation of the product for sale, she incurs a cost. Similar to our setting, there is a chance that a consumer forgoes purchasing a product that gives her positive utility, thinking that she will be able to purchase an even better product in the future; however, if she does not find a better product, then she might 
not end up purchasing any products throughout the selling season. Bernstein and Martínez-deAlbéniz (2016) study the retailer's optimal number of assortment changes in light of this tradeoff faced by the consumers. They model their problem as an optimal stopping problem since each consumer only purchases at most one product throughout the selling season. Since we allow each consumer to purchase multiple products throughout the selling season, we must use a different modeling technique since our problem cannot be cast as an optimal stopping problem. Bernstein and Martínez-de-Albéniz (2016) show that the retailer's optimal product rotation strategy is difficult to characterize and depends on whether the consumers are myopic or strategic, the cost of product rotation, and the capacity constraints.

To our knowledge, only our paper and Bernstein and Martínez-de-Albéniz (2016) consider assortment rotation strategies under dynamic consumer choice, i.e., when her purchase decisions in each period affect her purchase decisions in subsequent periods; in both papers, myopic consumers and strategic consumers are studied and compared. Recently, a few other papers have proposed and studied various reasons why a retailer may want to rotate assortments over the course of the selling season. Beyond differences in objectives, the key modeling difference between our work and the following is that ours includes strategic consumers and models dynamic consumer purchases, whereas the following do not. Caro et al. (2014) study the optimal timing of assortment changes when product attractiveness decays after the product is introduced. Davis et al. (2015) study which product, if any, to add to the assortment in each time period to maximize revenue throughout the selling season; they assume that at most one product can be added at a time, and once a product is added, it cannot be removed from the assortment. Bernstein et al. (2015) and Golrezaei et al. (2014) explore how a retailer can best offer dynamic, customized assortments given a customer's type and remaining inventory levels. Several papers have studied how a retailer can learn consumer demand by dynamically changing assortments (Ulu et al. 2012, Rusmevichientong et al. 2010, Sauré and Zeevi 2013, Caro and Gallien 2007, Farias and Madan 2011). There is also a vast literature on new product introduction timing which can influence a firm's assortment rotation strategy; however, technological advances are the main cause for the assortment rotations studied in most of this work (see, e.g., Ramachandran and Krishnan 2008, Krankel et al. 2006).

Our work also relates to a stream of research in the marketing literature on the effect of assortment size or variety on consumer demand and sales; however, unlike the other work referenced above, this stream of work does not consider assortment rotation strategies and rather focuses on the optimal single-period assortment size (see, e.g., Gourville and Soman 2005, Borle et al. 2005, Broniarczyk et al. 1998, Boatwright and Nunes 2001). 
Strategic consumers play a critical role in our model and results, and thus our work is also related to the broad literature on strategic consumers. Most of the operations management literature in this area has considered consumers to be strategic with respect to prices. In particular, strategic consumers face a tradeoff between purchasing a product at full price at the beginning of the selling season vs. purchasing the product at a discounted price at the end of the selling season subject to inventory availability. Shen and $\mathrm{Su}$ (2007) provide a good overview of this literature; more recent work in this area is Wu et al. (2015), Cachon and Swinney (2009), and Cachon and Swinney (2011) which study the fast-fashion setting. Li et al. (2014) and Soysal and Krishnamurthi (2012) also study consumers that are strategic with respect to prices, and they empirically estimate the percent of consumers who are strategic as opposed to myopic. Li et al. (2014) estimate that between $5.2 \%$ - $19.2 \%$ of consumers in the air-travel industry are strategic with respect to prices; Soysal and Krishnamurthi (2012) estimate that approximately $20 \%$ of customers of a fashion apparel retail chain are strategic towards the beginning of the selling season.

In our work, we consider consumers to be strategic with respect to their future valuation of products. There is considerably less literature studying this type of strategic consumer. One such stream of work considers a retailer selling a single product, and consumers face the tradeoff of purchasing the product of unknown valuation at the beginning of the selling season vs. purchasing the product towards the end of the selling season after realizing its valuation, subject to inventory availability; example papers include Swinney (2011) and DeGraba (1995). Our work differs from this stream of work in that we focus on a multi-product setting where consumers are strategic with respect to their future valuation of new, unique products. A few other papers have considered this type of strategic consumer, including Bernstein and Martínez-de-Albéniz (2016). Cachon et al. (2005) consider consumer choice across multiple retailers selling unique products when consumers are uncertain about their valuation of products offered by retailers that they have yet to visit; they identify the retailer's optimal (static) assortment in light of competition from other retailers. Lobel et al. (2016) consider consumers who are strategic with respect to their future valuation of new versions of a technology product, and they study how and when companies should develop and introduce each new version.

\section{Model}

During a given selling season, we suppose that a retailer (it) offers $N$ unique products, which we will index as $i=1, \ldots, N$, within a certain category for sale to a market of homogeneous consumers, whose size is normalized to 1 without loss of generality. We interpret the $N$ products as being 
"similar" and intuitively think of them as being identical in all respects except for style. Before the season begins, the retailer may choose either: $(i)$ a single assortment strategy where it offers all $N$ products throughout the entire selling season, or $(i i)$ a sequential assortment strategy where it offers each product sequentially over the course of the selling season; in the latter, we assume that the retailer divides the selling season into $N$ disjoint time periods, and each product is offered in exactly one period and each period has exactly one product.

For each assortment offered, the consumer (she) visits the retailer and decides which product(s), if any, to purchase from the current assortment. She makes these decisions throughout the season in order to maximize her expected utility. We emphasize that the total number of products purchased by the consumer over the entire selling season may be more than one. This is a key deviation from most choice and assortment models in the operations management literature.

We use a standard random utility model framework (Berry 1994) for the consumer's valuation of the retailer's products. In particular, we assume that her valuation comprises three additive components. The first component depends on the number of products that she has already purchased, $k=0,1, \ldots, N-1$, and is denoted $v(k)$. We interpret this first component as the consumer's base valuation of a typical product, and assume that $v$ is nonnegative and decreasing in $k$, reflecting diminishing utility from purchasing more products in the same category. That is, all else being the same, the consumer values the $k$ th product more than her $(k+1)$ th product. The second component is a random valuation shock that is realized only when she sees each product $i$, which we will denote as the random variable $X_{i}$. The third, and final, component is a disutility on the price $p$ paid for the product. Therefore, the consumer's utility from viewing the $i$ th product, assuming that she has already purchased $k<i$ products, is given by the random variable $v(k)+X_{i}-p$.

In our model, we further assume that:

(a) The retailer sells each of the $N$ products at the same price $p$ and incurs an identical cost for each product sold, which is assumed to be zero without loss of generality.

(b) The retailer has sufficient inventory to satisfy the maximum demand for each product.

(c) The consumer knows when assortment rotations occur and visits the retailer at negligible cost every time a new assortment is displayed.

(d) The consumer cannot return or exchange any purchased products.

Our model is purposefully stylized in order to focus on the impact of the retailer's assortment rotation strategy on revenue, as opposed to pricing, sourcing, or inventory decisions. Nevertheless, the first two assumptions are quite common in the literature (see, e.g., Bernstein and Martínezde-Albéniz (2016) for similar assumptions). Practically, there is somewhat anecdotal evidence that 
assumption (c) is representative for many flash sales and fast fashion retailers. For example, Rue La La, like many other flash sales retailers, sends emails to their entire customer base every time a new assortment is displayed on their site, and customers can simply click on the link in the email to visit the site and view the new assortment. In the fast fashion industry, it has been documented that customers visit Zara much more frequently than customers visit their more traditional retail competitors - an average of 17 visits per customer per year compared to just three (Kumar and Linguri 2006). Assumption (d) is consistent with the recent trend of online flash sales retailers offering "final sale" products that cannot be returned. Regardless of the retailer's actual return policy, this assumption is naturally satisfied when consumers start using the product as soon as it is purchased, i.e., the consumer wears her new pair of sandals the day after she buys them.

A common modeling assumption in the literature is to assume that consumers face budget constraints that only allow them to purchase up to a certain number of products throughout a given time period. We chose not to explicitly model budget constraints because for many of the product categories we are considering, the consumer's realistic budget would be quite large compared to the price of a product. Nevertheless, budget constraints can be accounted for in our model in a straightforward way: If the consumer's budget was such that she could not afford to purchase more than $B$ products throughout the selling season, we could model this by assuming that the random component of the consumer's valuation, $X_{i}$, has bounded support, in particular, so that $v(B)+X_{i}-p \leq 0$ with probability 1. In Section 4.2.3, we revisit this idea of a budget constraint and show how it naturally enters into an asymptotic analysis.

To summarize the model dynamics, the sequence of events is as follows:

1. Before the selling season begins, the retailer chooses either the single assortment strategy or the sequential assortment strategy.

2. For each new assortment,

(a) The retailer displays the product(s) in the assortment, and

(b) The consumer visits the store, realizes her valuation for each product in the assortment, and determines which product(s), if any, to purchase.

To illustrate with an example, Figure 2 depicts these dynamics when the retailer would like to sell six styles of sandals during the Spring/Summer season (in this example, March-August).

The decisions of the retailer and consumer form a two-stage game, and, as is standard in the analysis of such games, our analysis begins in Section 3 by studying the consumer's optimal purchase policy that maximizes her utility under both the single assortment and sequential assortment strategies. Under the sequential assortment strategy, we will analyze her decisions in two cases: 


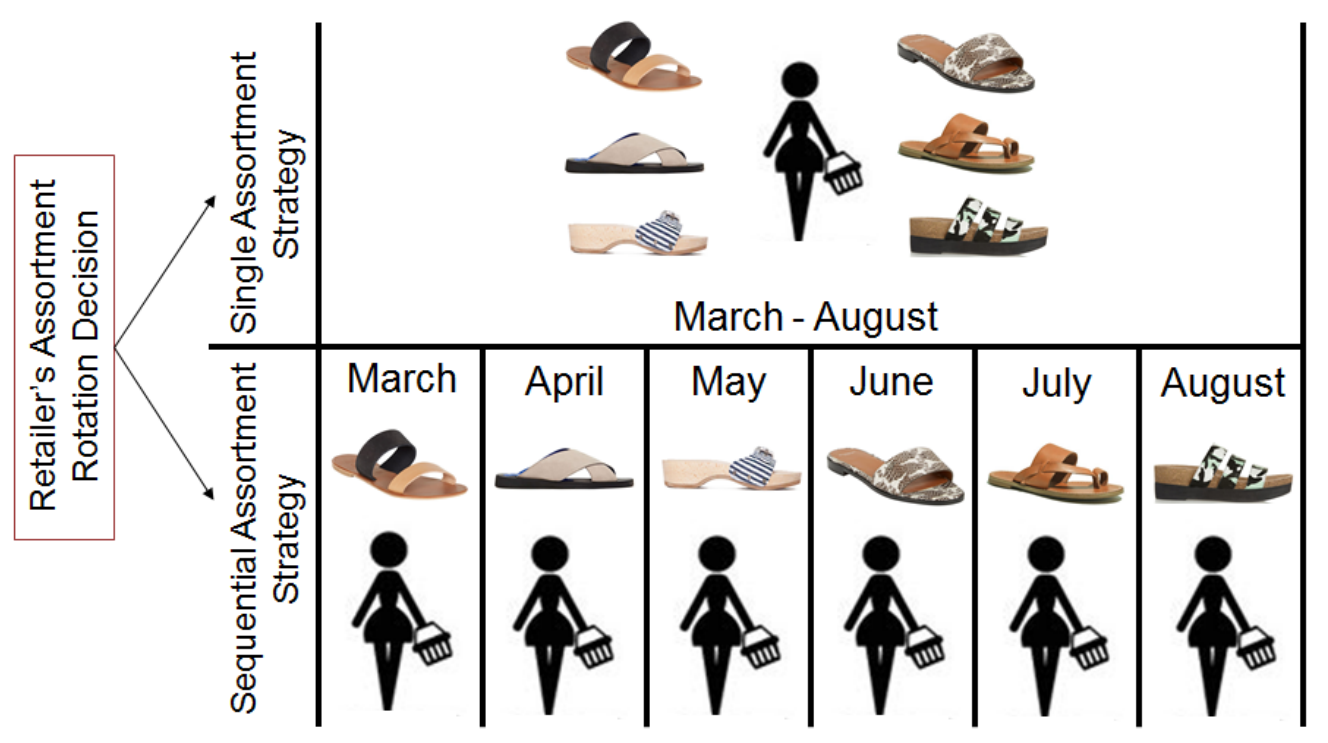

Figure 2 Model Dynamics for a Retailer Selling Six Styles of Sandals in the Spring/Summer Season.

when she is myopic, and when she is strategic. We defer a full description of each case to Section 3.2. Given the myopic and strategic consumer's optimal purchase policies, we will then study the retailer's optimal assortment rotation strategy and the value of concealment in Section 4.

\section{Notation}

We briefly digress to introduce some mathematical formalism and notation that we will adopt for the rest of this paper.

First, let $(\Omega, \mathcal{F}, \mathbb{P})$ be a probability space, and let $\mathbf{X}:=\left(X_{1}, \ldots, X_{N}\right)$ be random variables on this space, which are assumed to be integrable as well as independent and identically distributed (iid). Note that because of the iid assumption, at the beginning of the selling season, and before the retailer offers the first assortment, all products are identical in expectation to both the retailer and the consumer. To avoid going into technical minutiae, we will assume that $X_{1}, \ldots, X_{N}$ are continuous random variables, and denote their common cumulative distribution function (cdf) as $F$, and their common tail cdf as $\bar{F}:=1-F$. Let $\left\{\mathcal{F}_{i}\right\}_{i=0}^{N}$ represent the usual filtration associated with the random variables $X_{1}, \ldots, X_{N}$, so that $\mathcal{F}_{i}$ is the $\sigma$-field generated by the first $i$ random variables.

Second, let $\sigma(i), i=1, \ldots, N$ represent an ordering of the components of $\mathbf{X}$ in decreasing order such that $X_{\sigma(1)} \geq X_{\sigma(2)} \geq \ldots \geq X_{\sigma(N)}$. To be precise, $\sigma(\cdot)$ is a measurable function of $\mathbf{X}$, but we will suppress denoting its explicit dependence on $\mathbf{X}$ for notational brevity.

Third, $i=1, \ldots, N$ will typically be used to index the products, and $k=0, \ldots, i-1$ will typically be used to represent the number of products the consumer has already purchased. For ease of 
exposition, we will often choose to omit the ranges associated with $i$ and $k$ when it is clear from the context.

Finally, throughout the paper, we adopt the conventions that all unqualified (in)equalities involving random variables should be interpreted as holding $\mathbb{P}$-almost surely (a.s.), and all unqualified references to monotonicity properties (i.e., increasing/decreasing) refer to them in the weak sense.

All proofs can be found in Appendix A.

\section{Consumer's Optimal Policy}

We denote $\Pi^{1}$ and $\Pi^{N}$ as the set of all possible non-anticipating policies that could dictate a consumer's purchase behavior given that the retailer chooses the single assortment strategy or the sequential assortment strategy, respectively; non-anticipating policies preclude the consumer from using realization $X_{i}$ of future product $i$ 's valuation in her current purchase decisions. In both cases, a policy $\pi$ is $\{0,1\}^{N}$-valued, where $\pi_{i}=1$ is interpreted as "purchase product $i$ " and $\pi_{i}=0$ is interpreted as "do not purchase product $i$ ". A policy $\pi$ is in $\Pi^{1}$ if it is a measurable mapping from $\left(\Omega, \mathcal{F}_{N}\right)$ to $\left(\{0,1\}^{N}, 2^{\{0,1\}^{N}}\right)$, whereas a policy $\pi$ is in $\Pi^{N}$ if it is a process that is adapted to the filtration $\left\{\mathcal{F}_{i}\right\}_{i=0}^{N}$.

In the following subsections, we will derive the optimal (utility-maximizing) policies that maximize the consumer's utility under each of the retailer's assortment rotation strategies. Under the sequential assortment strategy, the optimal policy depends on whether or not consumers are myopic or strategic.

\subsection{Optimal Consumer Policy Under the Single Assortment Strategy}

Under the single assortment strategy, the retailer offers just one assortment with all $N$ products over the course of the selling season, and the consumer realizes her valuation for all $N$ products when she visits the store. Thus, the consumer has no uncertainty in product valuations at the time she must decide whether or not to purchase each product. The following lemma describes an optimal policy, $\pi^{1}$, for the consumer.

Lemma 1. Define $D^{1}$ as follows:

$$
D^{1}:= \begin{cases}0 & \text { if } v(0)+X_{\sigma(1)}<p, \text { and } \\ \sup \left\{j: v(j-1)+X_{\sigma(j)} \geq p\right\} & \text { otherwise. }\end{cases}
$$

When the retailer follows the single assortment strategy, the consumer maximizes her utility under the following policy, $\pi^{1}$, where $\forall i=1, \ldots, N$

$$
\pi_{i}^{1}:= \begin{cases}1 & \text { if } \sigma(i) \leq D^{1} \\ 0 & \text { otherwise }\end{cases}
$$


Lemma 1 implies that the consumer maximizes her total utility under the single assortment strategy by ordering the products in descending order based on her realized valuations, $\mathbf{X}$, and purchasing her favorite $D^{1}$ products such that product $\sigma\left(D^{1}\right)$ gives her positive utility and product $\sigma\left(D^{1}+1\right)$ does not. If no products give her positive utility, she will not purchase any of them; if all products give her positive utility, she will purchase all of them. Therefore, we interpret $D^{1}$ as the utility-maximizing (random) number of products purchased when the retailer follows the single assortment strategy. Below, we present another representation of $D^{1}$ :

$$
D^{1}=\sum_{i=1}^{N} \mathbb{1}_{\left\{X_{\sigma(i)} \geq p-v(i-1)\right\}} .
$$

It is routine to see that these two representations are equivalent.

\subsection{Optimal Consumer Policy Under the Sequential Assortment Strategy}

Under the sequential assortment strategy, the retailer shows $N$ assortments each with just one product, and the consumer realizes her valuation for each product only when its assortment is revealed. The retailer is effectively concealing a portion of its full product catalog from consumers. Thus, the consumer is uncertain about her valuations for each product in a subsequent assortment at the time she must decide whether or not to purchase the product in the current assortment. We emphasize that in this setting, every non-anticipating policy $\pi \in \Pi^{N}$ must map any set of realizations $X_{i}$ for products $i=1, \ldots, N$ and the order in which those realizations are revealed to the consumer's purchase decisions. We will denote product $i$ to be the $i$ th product offered in the selling season. Each new product or assortment may be equivalently interpreted as a "period" of a finite-horizon dynamic program of length $N$.

Unlike under the single assortment strategy, the consumer's optimal policy under the sequential assortment strategy depends on whether consumers are myopic or strategic. We address each case separately below.

3.2.1. Myopic Consumers. Myopic consumers are those who maximize immediate utility without concern of future assortments. For example, when a myopic consumer visits the retailer in period 1 to view the first assortment (i.e., first product), she realizes the random component of her valuation $X_{1}$ and makes a purchase decision without considering what utility she may gain in future assortments, i.e., from the (as-yet unrealized) values of $X_{2}, \ldots, X_{N}$.

In period $i=1, \ldots, N$, assuming that the consumer has already purchased $k=0, \ldots, i-1$ products, the myopic consumer solves the maximization problem $\max \left\{v(k)+X_{i}-p, 0\right\}$. Her total utility is therefore maximized by the threshold policy, $\pi^{\text {myopic }}$, where $\forall i=1, \ldots, N$

$$
\pi_{i}^{\text {myopic }}\left(k, X_{i}\right):= \begin{cases}1 & \text { if } X_{i} \geq m(k) \\ 0 & \text { otherwise }\end{cases}
$$


and where the threshold, $m(k)$, is defined as

$$
m(k):=p-v(k)
$$

Note that our definition of $\pi^{\text {myopic }}$ in (3) reflects that it only depends on $k$ and $X_{i}$, and is therefore a Markov policy. Furthermore, $m(k)$ is increasing in $k$ since we model $v(k)$ as a decreasing function of $k$ to reflect diminishing returns. Thus, the customer gets more selective as she purchases more products.

We now present an expression for the total number of products purchased by the myopic consumer over the entire selling season, which we denote as $D^{\text {myopic }}$, and which will be useful in our subsequent analysis. Define the stochastic process $\left\{Y_{i}\right\}_{i=0}^{N}$ as follows: Let $Y_{0}:=0$, and recursively define $Y_{i} \forall i=1, \ldots, N$ as

$$
Y_{i}:=Y_{i-1}+\mathbb{1}_{\left\{X_{i} \geq m\left(Y_{i-1}\right)\right\}} \cdot
$$

$Y_{i}$ can be interpreted as the number of products that the myopic consumer purchases through period $i$. Then, over the selling season, a myopic consumer purchases

$$
D^{\text {myopic }}=Y_{N}=\sum_{i=1}^{N} \mathbb{1}_{\left\{X_{i} \geq m\left(Y_{i-1}\right)\right\}} .
$$

Note that by construction, we necessarily have $Y_{i} \leq i$ for every $i=0, \ldots, N$.

\subsubsection{Strategic Consumers}

In contrast to myopic consumers, strategic consumers are those who seek to maximize their utility over the course of the entire selling season. For example, when a strategic consumer visits the retailer to view the first assortment, she realizes the random component of her valuation $X_{1}$ and makes a purchase decision considering what utility she believes that she may gain in future assortments, i.e., considering the (as-yet unrealized) values of $X_{2}, \ldots, X_{N}$. Therefore, we may express the strategic consumer's optimal policy as an optimal solution to the following Bellman equation: For $i=1, \ldots, N$ and $k=0, \ldots, i-1$, the strategic consumer's expected utility-to-go is

$$
\begin{aligned}
U_{i}(k) & =\mathbb{E}\left[\max \left\{v(k)+X_{i}-p+U_{i+1}(k+1), U_{i+1}(k)\right\}\right], \\
U_{N+1}(k) & =0 .
\end{aligned}
$$

The first term in the maximization, $v(k)+X_{i}-p+U_{i+1}(k+1)$, represents the consumer's (random) utility for purchasing product $i$ plus the expected future utility that she would earn given that she purchases product $i$. The second term in the maximization, $U_{i+1}(k)$, represents the consumer's expected future utility that she would earn given that she does not purchase product $i$. The expectation in (6) is over the consumer's random valuation of product $i$, namely, $X_{i}$. 
As in the myopic case, a threshold Markov policy, $\pi^{\text {strategic }}$, defined below, is optimal, where $\forall i=1, \ldots, N$ :

$$
\pi_{i}^{\text {strategic }}\left(k, X_{i}\right):= \begin{cases}1 & \text { if } X_{i} \geq s_{i}(k) \\ 0 & \text { otherwise. }\end{cases}
$$

Unlike the myopic case, where the thresholds $m(k), k=1, \ldots, N-1$ defined in (4) are simple, closed-form expressions of model parameters, the thresholds in the strategic case do not admit such simple expressions, but can be implicitly defined as:

$$
s_{i}(k):=p-v(k)-U_{i+1}(k+1)+U_{i+1}(k) .
$$

We note that $s_{N}(k)=m(k)$ for every $k$.

There is a representation of the total number of products purchased by a strategic consumer over the entire selling season, $D^{\text {strategic }}$, analogous to expression (5) for myopic consumers. Namely, define the stochastic process $\left\{Z_{i}\right\}_{i=1}^{N}$ as follows: Let $Z_{0}:=0$, and recursively define $Z_{i} \forall i=1, \ldots, N$ as

$$
Z_{i}:=Z_{i-1}+\mathbb{1}_{\left\{X_{i} \geq s_{i}\left(Z_{i-1}\right)\right\}}
$$

$Z_{i}$ can be interpreted as the number of products that the strategic consumer purchases through period $i$. Then, over the selling season, a strategic consumer purchases $D^{\text {strategic }}$ products, where

$$
D^{\text {strategic }}=Z_{N}=\sum_{i=1}^{N} \mathbb{1}_{\left\{X_{i} \geq s_{i}\left(Z_{i-1}\right)\right\}} .
$$

As before, we note that $Z_{i} \leq i$ for every $i=0, \ldots, N$.

The thresholds in (8) fully characterize the consumer's optimal policy. The next lemma collects several key properties of these thresholds, and characterizes how they relate to other parameters of our model.

Proposition 1. When the retailer follows the sequential assortment strategy, the thresholds $s_{i}(k)$ that determine the strategic consumer's optimal threshold policy satisfy the following properties:

(a) The thresholds increase in $k$ for every fixed $i$; that is, $s_{i}(k) \leq s_{i}(k+1)$.

(b) The thresholds decrease in $i$ for every fixed $k$; that is, $s_{i}(k) \geq s_{i+1}(k)$.

(c) The thresholds increase as the valuation distribution $F$ increases stochastically.

(d) The thresholds are bounded from above; specifically, $s_{i}(k) \leq m(k+N-i)$.

In Proposition 1, part (a) implies that the strategic consumer, like the myopic consumer, gets increasingly selective as she purchases more products - her $(k+1)$ th purchase has to meet a higher 
bar of attractiveness to her than her $k$ th purchase, given that she has already viewed $i$ products. Intuitively, since $v(k)$ is a decreasing function of $k$, the consumer experiences diminishing returns with each product she purchases and therefore is more selective. Part (b) implies that the strategic consumer gets less selective the closer she is to the end of the selling season. This is because she knows she'll be less likely to find a better product in a future assortment simply because there will be fewer products available and opportunities to purchase. For part (c), if the valuation distribution increases stochastically, products become generally more attractive to the consumer; being strategic, her expectation of future product valuations is more optimistic (higher), and she therefore becomes more selective in the current period about making a purchase. Finally, part (d) is mostly a technical property, but it reveals a simple sufficient condition for the strategic consumer's optimal purchasing behavior: It is optimal to purchase product $i$ if $X_{i} \geq p-v(k+N-i)$.

We can also derive key properties regarding the consumer's optimal utility.

Proposition 2. When the retailer follows the sequential assortment strategy, the strategic consumer's expected utility-to-go satisfies the following properties:

(a) Her expected utility-to-go decreases in $k$ for every $i$; that is, $U_{i}(k) \geq U_{i}(k+1)$.

(b) Her expected utility-to-go decreases in $i$ for every $k$; that is, $U_{i}(k) \geq U_{i+1}(k)$.

(c) Her expected utility-to-go is supermodular in $i$ and $k$; that is, $U_{i}(k)-U_{i+1}(k)$ is decreasing in $k$ for every $i$.

In Proposition 2, part (a) is a consequence of diminishing returns on each incremental product, i.e, $v(k)$ is a decreasing function of $k$; simply put, the more products she has already purchased, the lower her expected utility-to-go. To interpret parts (b) and (c), we first note that $U_{i}(k)-U_{i+1}(k)$ represents the decrease in the strategic consumer's expected utility-to-go if she has one less product to choose from. Thus $U_{i}(k)-U_{i+1}(k)$ may be interpreted as her "option value" in period $i$, i.e. the expected value she gains from having the option to purchase product $i$. We expect this quantity to be nonnegative (part (b)) since having the option to purchase one more product should be beneficial to the consumer. Part (c) further reveals that the value of this choice diminishes with each incremental product that she has already purchased. Intuitively, this is again a consequence of diminishing returns: All else being equal, each incremental product is less valuable, and hence the option value of being able to purchase that product also becomes progressively less valuable.

\section{Retailer's Optimal Assortment Rotation Strategy}

The retailer's objective is to maximize its revenue over the course of the selling season. To do this, the retailer has only one choice to make: Offer the $N$ products under the single or sequential 
assortment strategy. Because we have assumed that $\left\{X_{i}\right\}_{i=1}^{N}$ are i.i.d., the retailer is indifferent between the ordering of products in the sequential assortment strategy and thus this is not included as a decision.

Recall from Section 3.1 that $D^{1}$ represents the total number of products purchased by the consumer (i.e., the total demand) when the retailer chooses the single assortment strategy and the consumer follows optimal policy $\pi^{1}$. Further, we recall from Section 3.2.1 and Section 3.2.2 that for a consumer of type $t \in\{$ myopic, strategic $\}, D^{t}$ represents the total demand when the retailer chooses the sequential assortment strategy and the consumer follows the optimal policy $\pi^{t}$. Note that the randomness of demand is a consequence of the random component of the consumer's valuation of each product. The retailer's objective is therefore $\max \left\{\mathbb{E}\left[p D^{1}\right], \mathbb{E}\left[p D^{t}\right]\right\}=p \max \left\{\mathbb{E}\left[D^{1}\right], \mathbb{E}\left[D^{t}\right]\right\}$.

We formally define the value of concealment as $p\left(\mathbb{E}\left[D^{t}\right]-\mathbb{E}\left[D^{1}\right]\right)$, the difference in expected revenue between the sequential assortment strategy and single assortment strategy for the retailer. We note that when the value of concealment is positive, it is optimal for the retailer to choose the sequential assortment strategy. Conversely, when the value of concealment is negative, it is optimal for the retailer to choose the single assortment strategy. Finally, we note that the value of concealment (and therefore the retailer's optimal strategy) may differ depending on the consumer's type, $t$.

The following result characterizes the expected demand under the single assortment strategy.

Lemma 2. For each $k=1, \ldots, N$, let $B_{k}$ represent a binomial random variable such that $B_{k} \sim$ $\operatorname{Bin}(N, \bar{F}(m(k-1))$. Then, under the single assortment strategy,

(a) $\mathbb{P}\left(D^{1} \geq k\right)=\mathbb{P}\left(B_{k} \geq k\right)$, and

(b) Letting $m(k)$ be as defined in (4),

$$
\mathbb{E}\left[D^{1}\right]=\sum_{k=1}^{N} \sum_{i=k}^{N}\left(\begin{array}{c}
N \\
i
\end{array}\right) \bar{F}(m(k-1))^{i}[1-\bar{F}(m(k-1))]^{N-i} .
$$

The key idea of Lemma $2(\mathrm{a})$ is the observation that the event that $D^{1} \geq k$ is equivalent to the event that the $k$ th largest valuation, $X_{\sigma(k)}$, exceeds the threshold $m(k-1)$. In turn, for the latter to hold, there must be $k$ or more of the $X_{i}$ that exceed $m(k-1)$. Because $X_{i}$ are assumed to be i.i.d., this may be interpreted as $k$ or more "successes", where a single "success" is the event that a given $X_{i}$ exceeds $m(k-1)$, which occurs with probability $\bar{F}(m(k-1))$. Part (b) of Lemma 2 follows immediately from part (a) and the identity $\mathbb{E}\left[D^{1}\right]=\sum_{k=1}^{N} \mathbb{P}\left(D^{1} \geq k\right)$.

Since the consumer's optimal policy under the sequential assortment strategy depends on whether consumers are myopic or strategic, we address the expected demand in each case separately in 
the following subsections. For now, we state a preliminary result that holds for both myopic and strategic customers, which will be useful in our subsequent analysis.

Lemma 3. For $t \in\{$ myopic, strategic $\}, n=0, \ldots, N$ and $k=0, \ldots, N-n$, let $D_{k}^{t}(n)$ denote a random variable that represents the number of remaining products purchased by a type $t$ consumer who has already purchased $k$ products, and who has $n$ remaining assortments left to view. Then, for each $t, n$ and $k \leq N-n-1$, with probability 1 ,

$$
D_{k+1}^{t}(n) \leq D_{k}^{t}(n) \leq 1+D_{k+1}^{t}(n)
$$

The intuition behind this result is most clearly understood when customers are myopic. The result relates the remaining number of products purchased in the last $n$ periods for two different hypothetical customers (call them $A$ and $B$ ), where $A$ has purchased $k$ products and $B$ has purchased $k+1$ products prior to the $n$th period from the end of the horizon. Because we have assumed that the base valuation $v(k)$ decreases in $k$, we naturally have $D_{k+1}^{\text {myopic }}(n) \leq D_{k}^{\text {myopic }}(n)$, since $B$ is more selective than $A$. Lemma 3 shows that this increased selectiveness of $B$ is bounded: Even though $A$ purchases more than $B$, she does so by at most one unit. Furthermore, this same relationship holds even if customers are strategic.

In what follows, we compare the expected demand under the sequential assortment strategy with the expected demand under the single assortment strategy. Throughout this section, we will continue to denote period/product $i$ as the product that is sold in the $i$ th assortment, as we did in Section 3.2.

\subsection{Myopic Consumers}

To first gain some intuition on the impact of the myopic consumer's optimal purchase decisions on the retailer's expected demand under the sequential assortment strategy, we present an intermediate result below which establishes a monotonicity property on the myopic consumer's demand under pairwise interchanges of valuations.

Lemma 4. For any sequence of consumer valuations $X_{1}, \ldots, X_{N}$, let $j$ be any index such that $X_{j} \geq X_{j+1}$, assuming that it exists. Let $\widetilde{D}^{\text {myopic }}$ be the myopic consumer's demand under the same sequence of valuations with the sole exception that $X_{j}$ and $X_{j+1}$ are interchanged. Then, $\widetilde{D}^{\text {myopic }} \geq$ $D^{\text {myopic }}$.

The proof of Lemma 4 builds on the result in Lemma 3. The following proposition then applies Lemma 4 to derive the ordering of products that yield extremal demands.

Proposition 3. Under the sequential assortment strategy, a myopic consumer will 
(a) Purchase the smallest number of products if the products are sequenced in descending order of valuations, i.e., $X_{1} \geq X_{2} \geq \ldots \geq X_{N}$, compared to any other sequence of the same products.

(b) Purchase the largest number of products if the products are sequenced in ascending order of valuations, i.e., $X_{1} \leq X_{2} \leq \ldots \leq X_{N}$, compared to any other sequence of the same products.

Proposition 3 implies that consumers tend to buy more products when they are offered their least favorite products at the beginning of the selling season and their favorite products towards the end of the selling season. Recall from Section 3.2.1 that the myopic consumer's optimal policy, $\pi^{\text {myopic }}$, follows a threshold strategy such that the consumer will purchase product $i$ if and only if $X_{i}>m(k)$. Notice that this threshold, $m(k)$, is increasing in $k$, the number of products that the consumer has already purchased. Thus, myopic consumers are more selective as the selling season progresses and they purchase more products. Intuitively, the consumer purchases more products if the products that give her the largest $X_{i}$ are offered later in the selling season. The following example illustrates this point.

Example 1. Consider a retailer who sells two products (labeled $A$ and $B$ ) and who follows the sequential assortment strategy. The price of each product is $p=\$ 100$, and the (myopic) consumer's base valuations are $v(0)=\$ 90$ and $v(1)=\$ 80$. Consider the event that the consumer's realization of her random component of her valuation for product $A$ is $x_{A}=\$ 25$ and for product $B$ is $x_{B}=\$ 15$; she only observes $X_{i}=x_{i}$ when she is offered product $i \in\{A, B\}$.

Suppose the retailer offers product $A$ in the first assortment. The consumer realizes her valuation for the product $x_{A}=\$ 25>p-v(0)=\$ 10$, so she purchases product $A$. Then the retailer offers product $B$ in the second assortment, and the consumer realizes her valuation for the product $x_{B}=\$ 15<p-v(1)=\$ 20$, so she does not purchase product $B$.

Now suppose that instead, the retailer offers product $B$ in the first assortment. The consumer realizes her valuation for the product $x_{B}=\$ 15>p-v(0)=\$ 10$, so she purchases product $B$. Then the retailer offers product $A$ in the second assortment, and the consumer realizes her valuation for the product $x_{A}=\$ 25>p-v(1)=\$ 20$, so she also purchases product $A$.

Thus when the retailer offered the consumer's preferred product in the second assortment, the consumer purchased more products than when the retailer offered the consumer's preferred product in the first assortment.

Recall that the retailer does not know the consumer's valuations for any of the products at the beginning of the selling season. Thus, Proposition 3 is not suggesting that the retailer should offer products based on the realized values of $X_{1}, \ldots, X_{N}$; rather, it suggests that if the retailer happened to offer the products such that the consumer's realizations of $X_{1}, \ldots, X_{N}$ were presented 
in ascending order (i.e. with $X_{1} \leq X_{2} \leq \ldots \leq X_{N}$ ), the consumer would purchase at least as many products as she would have purchased had the products been offered in any other sequence.

Another reason that Proposition 3 is important is that it is a crucial step in allowing us to compare the retailer's demand under the single assortment strategy against the sequential assortment strategy when consumers are myopic.

THEOREm 1. With probability $1, D^{\text {myopic }} \geq D^{1}$. Therefore, if consumers are myopic, the retailer's value of concealment is always positive, i.e., the retailer maximizes its expected revenue by following the sequential assortment strategy.

Theorem 1 tells us that under the sequential assortment strategy, myopic consumers will never purchase fewer products than under the single assortment strategy; thus the retailer can expect greater demand, and therefore revenue, by following the sequential assortment strategy. This result is a consequence of the first part of Proposition 3: Under the sequential assortment strategy and given that consumers are myopic, the retailer will earn the least revenue if the products are offered in descending order. It turns out that this descending product ordering corresponds to the consumer's optimal decision process under the single assortment strategy; specifically, recall from Lemma 1 that under the single assortment strategy, the consumer will first order the products such that their realized valuations are in descending order, and then look at each product one-by-one in descending order to decide whether or not to buy the product. Intuitively, then, the consumer will purchase the same products under the single assortment strategy as she would purchase under the sequential assortment strategy when the products are offered in descending order. Since this is the product ordering that results in the least expected revenue for the retailer under the sequential assortment strategy, the retailer must always expect greater revenue from the sequential assortment strategy than from the single assortment strategy given consumers are myopic.

By offering products sequentially, the retailer is essentially introducing uncertainty in the consumer's future product valuations. It is precisely this uncertainty that induces the myopic consumer to purchase products that she otherwise may not have purchased had she realized her valuation for each product before having to make purchase decisions. This result highlights the positive value of concealment that a retailer can gain by rotating assortments throughout the course of the selling season.

\subsection{Strategic Consumers}

Before focusing solely on strategic consumers, we first compare the retailer's expected demand when consumers are strategic vs. when consumers are myopic. 
THEOREM 2. With probability $1, D^{\text {myopic }} \geq D^{\text {strategic }}$. Thus, the retailer's expected revenue when consumers are strategic is less than its expected revenue when consumers are myopic.

Theorem 2 tells us that strategic consumers are more selective in their product purchases than myopic consumers; interestingly, although their model setting is different than ours, Bernstein and Martínez-de-Albéniz (2016) also find that strategic consumers are more selective than myopic consumers. Thus, the retailer can always expect to earn greater revenue from myopic consumers than strategic consumers. Equivalently, the retailer's expected profit when facing myopic consumers serves as an upper bound for its expected profit when facing strategic consumers.

Next we would like to identify the retailer's optimal assortment rotation strategy (i.e., single or sequential) when consumers are strategic. As noted above, this entails analyzing the sign of the value of concealment, that is, we have to compare the relative values of $\mathbb{E}\left[D^{\text {strategic }}\right]$ and $\mathbb{E}\left[D^{1}\right]$. It turns out that this is generally a difficult relationship to determine, primarily because the consumer's optimal strategy is defined by solving the dynamic program in (6).

We present analytical and numerical results for the special case of $N=2$ in Section 4.2 .1 and $N=3$ in Section 4.2.2. In Section 4.2.2, we also illustrate the complexity of extending simple, analytical results to the case of general $N$. Finally, in Section 4.2.3 we present properties of the optimal assortment rotation strategy for the general $N$ case. We will show that the retailer's value of concealment is positive under certain conditions and negative in others. Interestingly, we find that the set of parameters that provide a retailer with a positive value of concealment tends to be those that describe products that are often found at flash sales and fast fashion retailers in practice. The results can help retail executives best exploit the value of concealment and determine which categories of products should be managed following the sequential assortment strategy and which categories of products should be managed following the single assortment strategy in the presence of strategic consumers.

4.2.1. Special Case: $N=2$. For the special case of just two products and when the retailer follows the sequential assortment strategy, the following describe the expected utility-to-go functions for the strategic consumer:

$$
\begin{aligned}
& U_{1}(0)=\mathbb{E}\left[\max \left\{v(0)+X_{1}-p+U_{2}(1), U_{2}(0)\right\}\right], \\
& U_{2}(0)=\mathbb{E}\left[\max \left\{v(0)+X_{2}-p, 0\right\}\right], \text { and } \\
& U_{2}(1)=\mathbb{E}\left[\max \left\{v(1)+X_{2}-p, 0\right\}\right] .
\end{aligned}
$$

The following theorem presents the retailer's optimal strategy for selling $N=2$ products when consumers are strategic. 
THEOREM 3. For $N=2$, the retailer's value of concealment is positive if and only if

$$
F(a)(F(b)-F(a)) \leq \bar{F}(c)(\bar{F}(b)-\bar{F}(c)),
$$

where $a:=p-v(0), c:=p-v(1)$, and $b:=a+\int_{a}^{c} \bar{F}(u) d u$. Thus if condition (14) holds, the retailer maximizes its revenue by following the sequential assortment strategy. Otherwise, it maximizes its revenue by following the single assortment strategy.

Note that when $N=2$, equations (11)-(13) illustrate that the strategic consumer must consider three purchasing thresholds: $p-v(0)-U_{2}(1)+U_{2}(0), p-v(0)$, and $p-v(1)$. Furthermore, we have $b=a+\int_{a}^{c} \bar{F}(u) d u=p-v(0)-U_{2}(1)+U_{2}(0)$. Therefore, Theorem 3 shows us that whether the retailer should choose the sequential or single assortment strategy depends on the probability density that falls between each of these three thresholds.

Although it is difficult to interpret condition (14), we note that if the valuation distribution $F$ is such that $X_{i} \geq a$ with probability $1, F(a)=0$ and condition (14) holds almost surely. This means that if the strategic consumer will buy at least one product almost surely - as is the case for many product categories that fast fashion and flash sales retailers sell - the retailer maximizes its expected demand (and therefore revenue) by following the sequential assortment strategy. The additional increase in revenue from using the sequential assortment strategy comes from the situation where the random component of the consumer's valuation for the product offered in the first assortment is smaller than that of the product offered in the second assortment, along the lines of Example 1.

To gain additional insights, we next present analytical results when $X_{i} \sim \operatorname{Uniform}(\alpha, \beta)$. We will consider the interesting set of parameters where $\alpha \leq p-v(0) \leq p-v(1) \leq \beta$. Note that the restriction $\alpha \leq p-v(0)$ implies that for some realizations of $X_{1}$ and $X_{2}$, the consumer will choose not to purchase either product $i=1,2$. The restriction $\beta \geq p-v(1)$ implies that for some realizations of $X_{1}$ and $X_{2}$, the consumer will purchase both products. The following result provides necessary and sufficient conditions under which the retailer maximizes its revenue by following the sequential assortment strategy.

Corollary 1. For $N=2, X_{i} \sim \operatorname{Uniform}(\alpha, \beta)$, and $\alpha \leq p-v(0) \leq p-v(1) \leq \beta$, the retailer's value of concealment is positive if and only if

$$
v(0)+v(1) \geq 2 p-\alpha-\beta .
$$

Thus if condition (15) holds, the retailer maximizes its revenue by following the sequential assortment strategy. Otherwise, it maximizes its revenue by following the single assortment strategy. 
Corollary 1 follows from Theorem 3 after algebraic manipulation of (14). Corollary 1 tells us that as long as the total "base" valuation that the strategic consumer would obtain from purchasing both products is large enough, the retailer maximizes its expected revenue by following the sequential assortment strategy. By recognizing that $\mathbb{E}\left[X_{i}\right]=\frac{\alpha+\beta}{2}$ and rearranging terms in condition (15), condition (15) can be re-written as

$$
\left(v(0)+\mathbb{E}\left[X_{1}\right]-p\right)+\left(v(1)+\mathbb{E}\left[X_{2}\right]-p\right) \geq 0 .
$$

In other words, as long as the consumer's expected total valuation from purchasing both products is non-negative, the retailer maximizes its revenue by following the sequential assortment strategy; note that this is less restrictive than requiring her expected valuation from purchasing each product to be non-negative.

Figure 3 shows a heat map of the value of concealment, expressed as a percentage of the retailer's expected revenue from following the single assortment strategy, for the case where $p=1, X_{i} \sim$ $\operatorname{Uniform}(0,1) ; i=1,2$ and for different values of $v(0) \in[0,1], v(1) \in[0, v(0)]$. In other words, Figure 3 illustrates the percent increase in demand when the retailer follows the sequential assortment strategy compared to the single assortment strategy. The yellow region of the heat map represents values of $v(0)$ and $v(1)$ where the value of concealment is positive, i.e., the retailer earns more revenue by following the sequential assortment strategy; the brighter the shade of yellow, the greater the value of concealment. In contrast, the red region of the heat map represents values of $v(0)$ and $v(1)$ where the value of concealment is negative, i.e., the retailer earns less revenue by following the sequential assortment strategy. Note that for $p=1, \alpha=0$, and $\beta=1$, condition (15) becomes $v(0)+v(1) \geq 1$ which is the boundary line in Figure 3 that separates the yellow and red regions.

Figure 3 illustrates that for some values of $v(0)$ and $v(1)$, the value of concealment can be substantial in both directions. In particular, we observe that the retailer can earn substantially more revenue - as much as a $7 \%$ increase in revenue for this set of parameters - simply by following the sequential assortment strategy as opposed to the single assortment strategy. Conversely, for other values of $v(0)$ and $v(1)$, the retailer may earn substantially less revenue - as much as a $9 \%$ decrease in revenue - by following the sequential assortment strategy compared to the single assortment strategy. To help the retailer understand when it should follow each assortment rotation strategy, we will focus on two extremes to help gain some intuition.

First, we look at the case when $v(0)=1$. This is the area of Figure 3 that is brightest yellow and generally where the retailer has the greatest value of concealment. In this case, the consumer will 


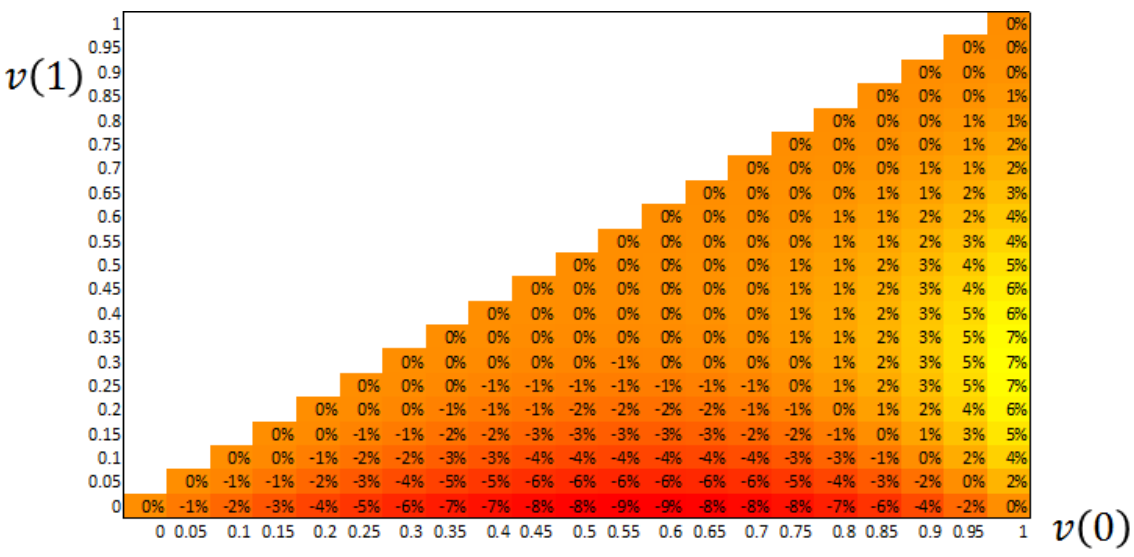

Figure 3 Value of concealment as a percentage of expected revenue from the single assortment strategy: $p=1$ and $X_{i} \sim \operatorname{Uniform}(0,1) ; i=1,2$

certainly purchase at least one product regardless of which assortment rotation strategy the retailer follows. The increase in expected demand comes from the situation where after viewing the first product and realizing her valuation, the consumer believes that she will not like the second product as much as the first; in this case she may decide to purchase the first product. Subsequently, she realizes her valuation for the second product which she ends up liking even more than the first, so much so that she may also purchase the second product. Had the retailer offered both products in a single assortment, the consumer may have only purchased her favorite product. Similar intuition was developed in Example 1.

Next, we look at the case when $v(1)=0$. This is the area of Figure 3 that is brightest red and generally where the retailer has the lowest value of concealment. In this case, the consumer will purchase at most one product regardless of which assortment rotation strategy the retailer follows. The decrease in expected demand comes from the situation where after viewing the first product and realizing her valuation, the consumer believes that she will like the second product more than the first; in this case she decides not to purchase the first product. Subsequently, she realizes her valuation for the second product which she ends up not liking as much as the first, so much so that she may also decide not to purchase the second product. Had the retailer offered both products in a single assortment, the consumer may have purchased her favorite product.

The intuition we have developed here extends beyond just the special case of $N=2$ products and where $X_{i} \sim \operatorname{Uniform}(0,1)$. In Appendix B, we present numerical results when $N=2$ and $X_{i} \sim \operatorname{Normal}\left(\mu=0.5, \sigma^{2}=\frac{1}{12}\right)$ and show that our intuition is identical for this special case, too. Furthermore, our results in Section 4.2.3 suggest that similar intuition extends to the case of more general $N$. 
Interestingly, for many of the product categories that fast fashion and flash sales retailers sell, such as sandals and dresses, consumers often purchase multiple products. Our results suggest that for categories such as these, retailers can realize a positive value of concealment from introducing uncertainty in future product valuations by offering products sequentially. Occasionally, fast fashion and flash sales retailers sell products in categories where it is expected that a consumer will purchase at most one product; for example, Rue La La has sold engagement rings via the sequential assortment strategy. In this case, our results suggest that product concealment has a negative value, and therefore Rue La La could potentially generate more demand by instead offering all engagement rings in a single assortment. Following a single assortment strategy eliminates the consumer's uncertainty about her valuation of the entire catalog of engagement rings for sale, and maximizes the likelihood that she will purchase her favorite ring. Thus our results provide guidance to retailers regarding which product categories to sell via the sequential versus single assortment strategies. Specifically, our intuition suggests that for product categories where consumers tend to purchase multiple products, the value of concealment is positive and retailers should follow the sequential assortment strategy; for product categories where consumers tend to purchase very few products, the value of concealment is negative and retailers should follow the single assortment strategy.

4.2.2. Special Case: $N=3$. To develop intuition for the case of $N=3$, we consider the special case where $X_{i} \sim \operatorname{Uniform}(0,1)$. We aim to find necessary and sufficient conditions on the parameters $(x, y, z):=(p-v(0), p-v(1), p-v(2))$ to establish that $\mathbb{E}\left[D^{\text {strategic }}\right] \geq \mathbb{E}\left[D^{1}\right]$ and vice versa. The following proposition captures the main result:

Proposition 4. Consider the special case where $N=3$ and $X_{i} \sim \operatorname{Uniform}(0,1)$. For a given trivariate, 7th degree, polynomial, $g(x, y, z)$, the value of concealment is positive if and only if $g(x, y, z) \geq 0$.

Unfortunately, we had to resort to brute force computation in order to construct the trivariate polynomial $g$ used in the proof of Proposition 4, and were not able to find a simple representation for $g$. The full representation of $g$ is listed in the proof of Proposition 4 in the appendix. The analysis of this special case suggests that it is unlikely that there are simple necessary and sufficient conditions to characterize the sign of the value of concealment, even for the case of $N=3$.

Figure 4 illustrates the boundary constructed from the polynomial $g$ where the expected demand from strategic consumers under the sequential assortment strategy is equal to the expected demand under the single assortment strategy. Visual inspection indicates that it has a rather complicated overall shape. 


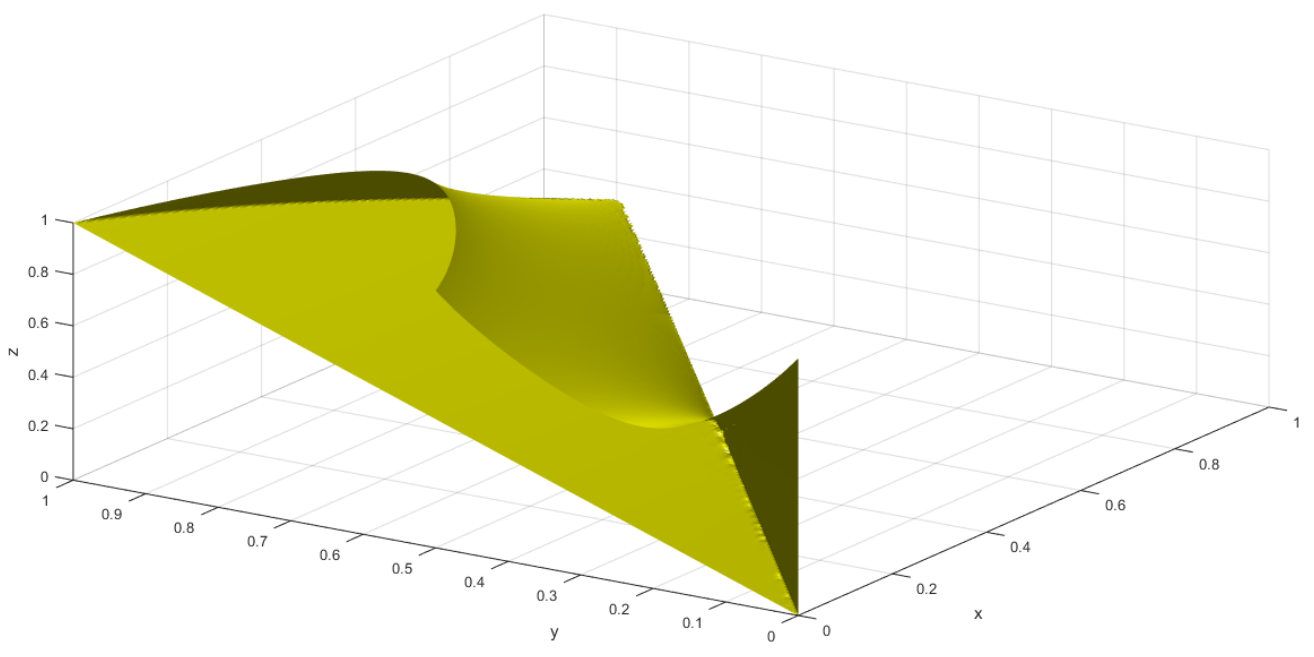

(a)

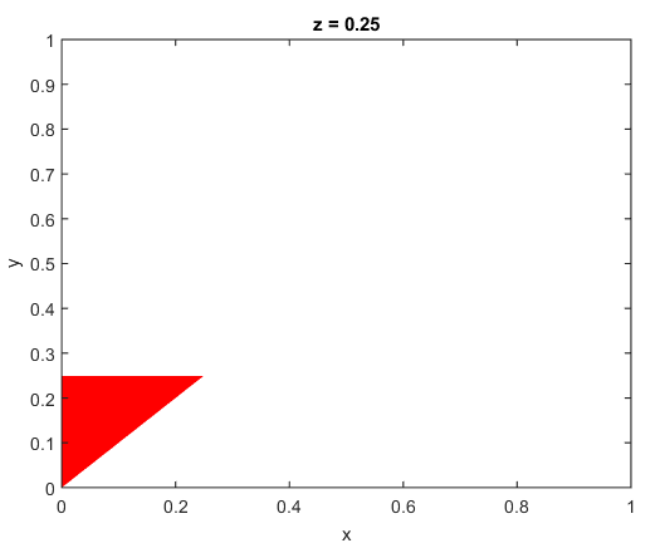

(b)

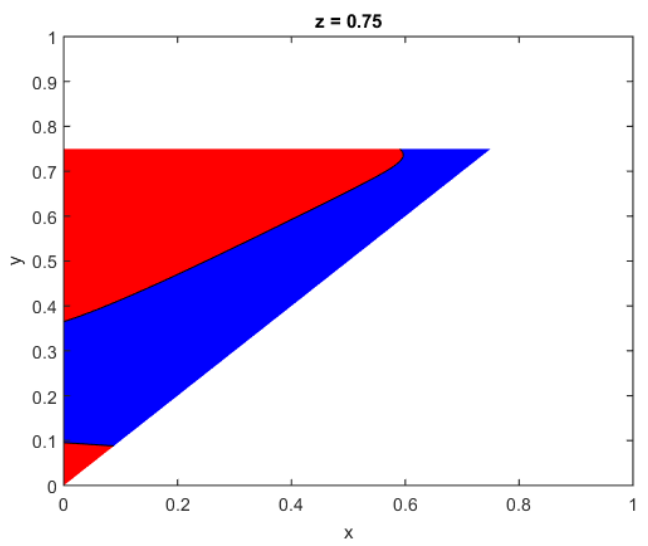

(d)

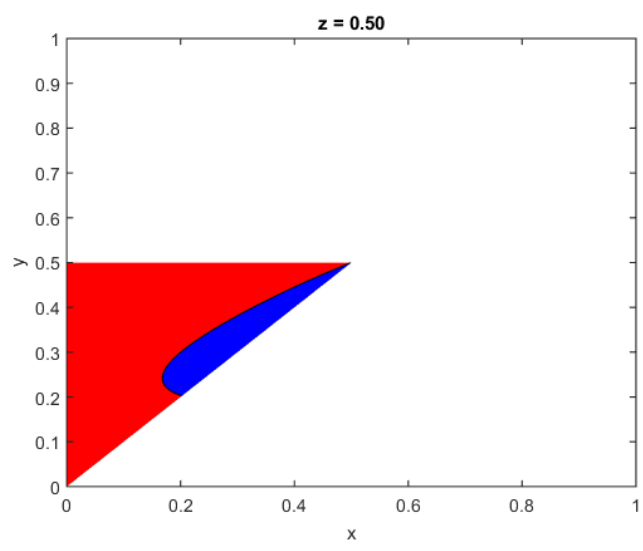

(c)

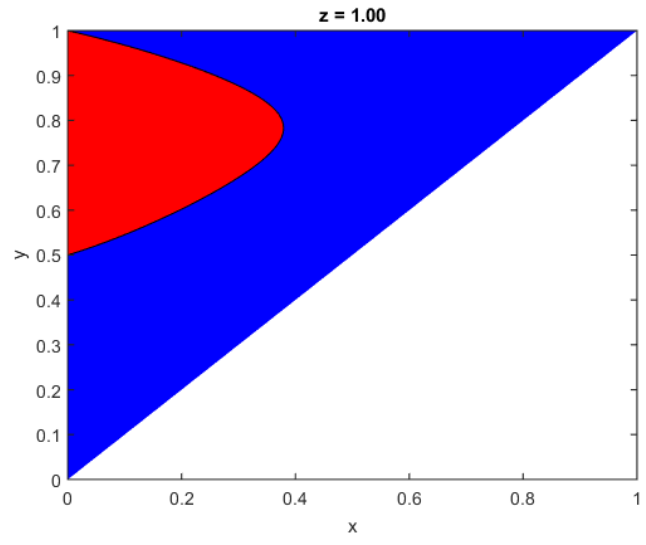

(e)

Figure 4 For the special case where $N=3$ and $X_{i} \sim U$ niform $(0,1)$ : (a) 3D plot of boundary on which the value of concealment is zero. (b) - (e) Ranges of $(x, y)$ for which the value of concealment is strictly positive (blue) and strictly negative (red), for various values of $z$. 
4.2.3. General $N$ : For the case of general $N$, as the results in Section 4.2 .2 suggest, it would be very difficult - if not impossible - to develop simple, necessary and sufficient conditions to determine when the value of concealment is positive or negative. Therefore, we focus on developing sufficient conditions for the retailer's optimal assortment rotation strategy and continue to build our intuition. We begin with the following theorem.

Theorem 4. For any $N \geq 2$,

(a) If $X_{i} \geq m(N-2)$ with probability 1 , then the value of concealment is positive.

(b) If $X_{i} \leq m(1)$ with probability 1 , then the value of concealment is negative.

We note several points about Theorem 4. First, at least directionally, the intuition that was developed for the case where $N=2$ continues to hold more generally. Specifically, for product categories where strategic consumers tend to purchase multiple products, the retailer will maximize demand by offering products sequentially; this is suggested by part (a) of Theorem 4. Conversely, for product categories where strategic consumers tend to purchase just a few products, the retailer will maximize demand by offering products in a single assortment; this is suggested by part (b) of Theorem 4.

Second, even though the sufficient conditions for the theorem seem stringent, one can apply a continuity argument to arrive at weaker sufficient conditions - namely, the theorem would hold even if the valuation vectors $v$ do not satisfy Theorem 4 exactly, but are close enough.

Third, building on the intuition of this theorem, one might be tempted to conjecture that the value of concealment should be higher if the random valuation shock, $X_{i}$, is stochastically larger. Unfortunately, this result does not hold in general. One only needs to look at the case of $N=2$ in Section 4.2.1 (for which $\mathbb{E}\left[D^{\text {strategic }}\right]$ and $\mathbb{E}\left[D^{1}\right]$ have simple expressions) to find a counterexample.

We now consider the asymptotic demand as the number of products offered goes to infinity. To emphasize, for this part of our analysis we interpret the number of products, $N$, not as being a fixed parameter, but as varying. Broadly speaking, the following theorem shows that the demand that the retailer experiences when using either the single assortment or sequential assortment strategy are asymptotically equivalent as $N$ goes to infinity, for both myopic and strategic consumers.

TheOREm 5. Recall the definitions of $D^{t}$ for each $t \in\{1$, myopic, strategic $\}$.

(a) Suppose that $\lim _{N \rightarrow \infty} \mathbb{P}\left(X_{i} \geq p-v(N-1)\right)=\delta>0$. Then, as $N \rightarrow \infty, D^{t} / N \stackrel{\text { a.s. }}{\rightarrow} \delta$.

(b) Define $N_{0}:=\sup \left\{N \geq 1: \mathbb{P}\left(X_{i} \geq p-v(N-1)\right)>0\right\}$. Suppose that $N_{0}$ is finite. Then, as $N \rightarrow$ $\infty, D^{t} \stackrel{a . s .}{\rightarrow} N_{0}$. 
The first part of the theorem shows that if there is some probability $\delta>0$ such that the random component of the consumer's valuation exceeds her limiting myopic threshold, then for large $N$, the quantity that she purchases is asymptotically linear in $N$ regardless of the assortment rotation strategy the retailer follows or the type of consumer (myopic or strategic). This tells us that as $N \rightarrow \infty$, the increase (or decrease) in demand that the retailer can induce by offering products sequentially becomes negligible, i.e., the value of concealment is negligible. To see the intuition behind this result, recall that a key difference between the sequential and single assortment strategies is that the consumer faces uncertainty in future product valuations in the sequential assortment strategy. When $N \rightarrow \infty$, this uncertainty is essentially eliminated because the consumer becomes more and more certain that she will eventually find a product that gives her the utility she's looking for, simply because she's able to choose from more products. Without this uncertainty, expected demand is the same under the sequential and single assortment strategies.

The second part of the theorem shows that if the random component of her valuation has some probability of exceeding the threshold for $N_{0}$ products, but is almost surely below the threshold for $N_{0}+1$ products, then asymptotically, as the number of products $N$ increases to infinity, she will purchase $N_{0}$ products with probability 1 . Intuitively, $N_{0}$ acts as the consumer's budget for purchases. While this result is rather intuitive if the consumer is myopic, it is not obvious that it would hold if she is strategic: In particular, given a very long selling season, a strategic consumer could, in principle, apply such stringent thresholds for periods that are very far away from the end of the selling season that one might intuit that she has some positive probability of purchasing strictly less than $N_{0}$. Our result shows that this occurs with zero probability. Similar to part (a), part (b) of Theorem 5 also suggests that as $N \rightarrow \infty$, the consumer's uncertainty in future product valuations under the sequential assortment strategy becomes inconsequential, and thus the retailer induces the same demand under the sequential and single assortment strategies. Theorem 5 highlights the role of uncertainty in future product valuations that the sequential assortment strategy introduces.

\section{Managerial Insights and Conclusion}

As more and more retailers are choosing to frequently rotate their assortments, it is important to understand the implications of such frequent assortment rotation on the consumer's purchase decisions. Our work identifies and studies a new reason for retailers to have assortment rotations they may be able to obtain a positive value of concealment and thereby generate additional sales. In this paper, we developed a model of a consumer's purchase decisions throughout a selling season 
when she considers purchasing multiple products in the same category, and applied this model to gain an understanding around when this value of concealment is positive and vice versa.

We studied the impact of frequent assortment rotations on both myopic and strategic consumers; here, we expand upon the limited research on strategic consumers where consumers are "strategic" with respect to the consumer's unknown future product valuations. We found that under certain conditions and given a fixed number of products, the retailer can earn more revenue simply by increasing the number of assortments offered throughout the season. Our results and intuition provide guidance to retail executives regarding which product categories should be managed following the sequential vs. single assortment strategies. Specifically, for myopic consumers, the retailer always has a positive value of concealment and maximizes its expected revenue by following the sequential assortment strategy; in other words, the consumer's uncertainty in future product valuations causes her to purchase at least as many products when she is offered products sequentially as she would have purchased had she been offered products in a single assortment. Furthermore, myopic consumers will always purchase at least as many products as strategic consumers, regardless of the retailer's assortment rotation strategy.

For strategic consumers, we identify conditions under which the value of concealment is positive and vice versa. Our analytical and numerical results help guide our intuition that for product categories where consumers are very likely to buy many products within the category throughout the selling season, the uncertainty in future product valuations that the sequential assortment strategy introduces can cause the strategic consumer to purchase more products than if she had been offered them simultaneously; in this case, the retailer has a positive value of concealment and maximizes its revenue by following the sequential assortment strategy. Corroborating our intuition, for many of the product categories that fast fashion and flash sales retailers sell - such as fashionable apparel, accessories, and children's toys - consumers are very likely to buy many products within the category throughout the selling season. Some restaurants are even employing a type of sequential assortment strategy; for example, some sushi restaurants serve unique sushi rolls on a conveyor belt that passes in front of customers. Customers must decide whether or not they want to purchase the sushi roll before observing subsequent sushi rolls that will be added to the conveyor belt later in the meal. Since customers are likely to purchase many sushi rolls over the course of a dinner, our results suggest that customers may end up purchasing more sushi at such a restaurant compared to a sushi restaurant where customers order from a fixed menu.

In contrast, when consumers consider buying at most one or very few products within the category throughout the season, the uncertainty in future product valuations that the sequential 
assortment strategy introduces can cause the strategic consumer not to purchase a product that she would have attained positive utility from; in this case, the retailer's value of concealment is negative, and it instead maximizes its revenue by following the single assortment strategy. Examples of such product categories include automobiles, home appliances, and even some apparel and accessories categories (e.g. engagement rings).

Our present paper opens up several potential avenues for future work. First, since our main results depend on whether a consumer is myopic or strategic, it is important for retailers to be able to classify their consumers into one of these two types. We believe there could be interesting empirical work done in partnership with retailers to help address this challenge. Another interesting extension to our work would be to relax the assumption that all products have the same margin and include the retailer's decision of which product to sell in each assortment (i.e., the order in which to offer products in the sequential assortment strategy). Given the difficulties in attaining analytical boundary conditions for the general $N$ case even with this assumption, this open question is likely challenging to solve analytically. Finally, we believe it would be interesting to include behavioral factors into this line of research. For example, customers may experience a dis-utility of regret if they forgo purchasing a product in hopes of finding a better one in a subsequent assortment, and then end up not finding a better product.

\section{Acknowledgments}

We would like to thank David Simchi-Levi, Victor Martínez-de-Albéniz, and Özalp Özer for several very helpful and insightful discussions shaping this research. We would also like to thank Andrew Marder for his assistance with numerical simulations.

\section{References}

Bernstein, F., A. G. Kök, L. Xie. 2015. Dynamic Assortment Customization with Limited Inventories. Manufacturing $\& 3$ Service Operations Management 17(4) 538-553.

Bernstein, F., V. Martínez-de-Albéniz. 2016. Dynamic Product Rotation in the Presence of Strategic Customers. Management Science. Forthcoming.

Berry, S. T. 1994. Estimating Discrete-Choice Models of Product Differentiation. The RAND Journal of Economics 242-262.

Boatwright, P., J. Nunes. 2001. Reducing Assortment: An Attribute-Based Approach. Journal of Marketing 65(3) 50-63.

Borle, S., P. Boatwright, J. Kadane, J. Nunes, G. Shmueli. 2005. The Effect of Product Assortment Changes on Customer Retention. Marketing Science 24(4) 616-622. 
Broniarczyk, S., W. Hoyer, L. McAlister. 1998. Consumers' Perceptions of the Assortment Offered in a Grocery Category: The Impact of Item Reduction. Journal of Marketing Research 35(2) 166-176.

Cachon, G. P., R. Swinney. 2009. Purchasing, Pricing, and Quick Response in the Presence of Strategic Consumers. Management Science 55(3) 497-511.

Cachon, G. P., R. Swinney. 2011. The Value of Fast Fashion: Quick Response, Enhanced Design, and Strategic Consumer Behavior. Management Science 57(4) 778-795.

Cachon, G. P., C. Terwiesch, Y. Xu. 2005. Retail Assortment Planning in the Presence of Consumer Search. Manufacturing \& Service Operations Management 7(4) 330-346.

Caro, F., J. Gallien. 2007. Dynamic Assortment with Demand Learning for Seasonal Consumer Goods. Management Science 53(2) 276-292.

Caro, F., V. Martínez-de-Albéniz. 2012. Product and Price Competition with Satiation Effects. Management Science 58(7) 1357-1373.

Caro, F., V. Martínez-de-Albéniz, P. Rusmevichientong. 2014. The Assortment Packing Problem: Multiperiod Assortment Planning for Short-Lived Products. Management Science 60(11) 2701-2721.

Chang, T., D. Gianera, L. Huang, A. Inderst, D. Isaacson, C. Jian, S. Mahamkali, C. Storey, L. Vasilescu, T. Williams. 2016. Global Fashion. Tech. rep., Macquarie Research.

Davis, J. M., H. Topaloglu, D. P. Williamson. 2015. Assortment Optimization Over Time. Operations Research Letters 43(6) 608-611.

DeGraba, P. 1995. Buying Frenzies and Seller-Induced Excess Demand. The RAND Journal of Economics 26(2) 331-342.

Dishman, L. 2012. Nordstrom + Topshop: Another Retail Odd Couple Makes a Grab for New Customers. Forbes. Accessed online May 2016.

Farias, V., R. Madan. 2011. The Irrevocable Multiarmed Bandit Problem. Operations Research 59(2) 383-399.

Fisher, Marshall, Ananth Raman. 1996. Reducing the cost of demand uncertainty through accurate response to early sales. Operations research 44(1) 87-99.

Golrezaei, N., H. Nazerzadeh, P. Rusmevichientong. 2014. Real-Time Optimization of Personalized Assortments. Management Science 60(6) 1532-1551.

Gourville, J., D. Soman. 2005. Overchoice and Assortment Type: When and Why Variety Backfires. Marketing Science 24(3) 382-395.

Kapner, S. 2015. Gap to Test Fast Fashion Model in Select Stores. The Wall Street Journal. Accessed online May 2016.

Krankel, R. M., I. Duenyas, R. Kapuscinski. 2006. Timing Successive Product Introductions with Demand Diffusion and Stochastic Technology Improvement. Manufacturing 83 Service Operations Management 8(2) 119-135. 
Kumar, N., S. Linguri. 2006. Fashion Sense. Business Strategy Review 17(2) 80-84.

Li, J., N. Granados, S. Netessine. 2014. Are Consumers Strategic? Structural Estimation from the Air-Travel Industry. Management Science 60(9) 2114-2137.

Lobel, I., J. Patel, G. Vulcano, J. Zhang. 2016. Optimizing Product Launches in the Presence of Strategic Consumers. Management Science Forthcoming.

Ramachandran, K., V. Krishnan. 2008. Design Architecture and Introduction Timing for Rapidly Improving Industrial Products. Manufacturing \& Service Operations Management 10(1) 149-171.

Rusmevichientong, P., Z. M. Shen, D. B. Shmoys. 2010. Dynamic Assortment Optimization with a Multinomial Logit Choice Model and Capacity Constraint. Operations Research 58(6) 1666-1680.

Sauré, D., A. Zeevi. 2013. Optimal Dynamic Assortment Planning with Demand Learning. Manufacturing ES Service Operations Management 15(3) 387-404.

Shen, Z. M., X. Su. 2007. Customer Behavior Modeling in Revenue Management and Auctions: A Review and New Research Opportunities. Production and Operations Management 16(6) 713-728.

Soysal, G., L. Krishnamurthi. 2012. Demand Dynamics in the Seasonal Goods Industry: An Empirical Analysis. Marketing Science 31(2) 293-316.

Swinney, R. 2011. Selling to Strategic Consumers When Product Value Is Uncertain: The Value of Matching Supply and Demand. Management Science 57(10) 1737-1751.

Ulu, C., D. Honhon, A. Alptekinoğlu. 2012. Learning Consumer Tastes Through Dynamic Assortments. Operations Research 60(4) 833-849.

Wu, S., Q. Liu, R. Q. Zhang. 2015. The Reference Effects on a Retailer's Dynamic Pricing and Inventory Strategies with Strategic Consumers. Operations Research 63(6) 1320-1335. 


\section{APPENDIX A: Proofs}

Proof of Lemma 1. Suppose that $\left\{X_{i}\right\}_{i=1}^{N}$ are given. Further suppose that if the consumer acts optimally, she purchase $\ell$ products, where $\ell \in\{0, \ldots, N\}$. Given that she purchases $\ell$ products, it is obvious that her utility-maximizing choice of $\ell$ products are the $\ell$ products with the highest valuations, i.e., $X_{\sigma(1)}, \ldots, X_{\sigma(\ell)}$. Therefore, the consumer's problem can be written as

$$
\max _{\ell=0, \ldots, N}\left\{\sum_{i=1}^{\ell}\left(v(i-1)+X_{\sigma(i)}-p\right)\right\} .
$$

We follow common convention and define the value of any empty sums to be zero. Now, let $D^{1}$ be as defined in the statement of the lemma. This implies that

$$
v(i-1)+X_{\sigma(i)} \geq p \quad \forall i=0, \ldots, D^{1},
$$

and

$$
v(i-1)+X_{\sigma(i)}<p \quad \forall i=D^{1}+1, \ldots, N
$$

Consider any $\ell=0, \ldots, D^{1}$. By $(17)$,

$$
\sum_{i=1}^{D^{1}}\left(v(i-1)+X_{\sigma(i)}-p\right)-\sum_{i=1}^{\ell}\left(v(i-1)+X_{\sigma(i)}-p\right)=\sum_{i=\ell+1}^{D^{1}} \underbrace{\left(v(i-1)+X_{\sigma(i)}-p\right)}_{\geq 0} \geq 0 .
$$

Next, consider any $\ell=D^{1}+1, \ldots, N$. By $(18)$,

$$
\sum_{i=1}^{D^{1}}\left(v(i-1)+X_{\sigma(i)}-p\right)-\sum_{i=1}^{\ell}\left(v(i-1)+X_{\sigma(i)}-p\right)=-\sum_{i=D^{1}+1}^{\ell} \underbrace{\left(v(i-1)+X_{\sigma(i)}-p\right)}_{\leq 0} \geq 0 .
$$

Hence, her utility is maximized by choosing $\ell=D^{1}$, that is, only purchasing products $i$ that have $\sigma(i) \leq D^{1}$. Q.E.D.

Proof of Proposition 1. Throughout this proof, we will use the more compact notation $s_{i}^{k}:=s_{i}(k)$. We first prove a recursive expression for $s_{i}^{k}$. Note that we can rewrite the Bellman equation (6) as

$$
U_{i}(k)=U_{i+1}(k)+\mathbb{E}\left[\left(X_{i}-s_{i}^{k}\right)_{+}\right]=U_{i+1}(k)+\int_{s_{i}^{k}}^{\infty} \bar{F}(u) d u,
$$

where $(z)_{+}:=\max \{z, 0\}$, and the final equality is a result of the identity $\mathbb{E}\left[(X-x)_{+}\right] \equiv \int_{x}^{\infty} \bar{F}(u) d u$. Now consider

$$
\begin{array}{rlr}
s_{i-1}^{k}-s_{i}^{k} & =U_{i}(k)-U_{i}(k+1)-U_{i+1}(k)+U_{i+1}(k+1) & {[\operatorname{By}(8)]} \\
& =\int_{s_{i}^{k}}^{\infty} \bar{F}(u) d u-\int_{s_{i}^{k+1}}^{\infty} \bar{F}(u) d u & {[\text { By (19)] }} \\
& =\int_{s_{i}^{k}}^{s_{i}^{k+1}} \bar{F}(u) d u &
\end{array}
$$


Therefore,

$$
s_{i-1}^{k}=s_{i}^{k}+\int_{s_{i}^{k}}^{s_{i}^{k+1}} \bar{F}(u) d u
$$

We prove part (a) by backward induction on $i$. For $i=N$, we have $s_{N}^{k}=s_{N}(k)=m(k)$, which is increasing in $k$ by the assumption that $v(k)$ is a decreasing function of $k$. Inductively suppose that for some $i, s_{i}^{k}$ is increasing in $k$ for every $k$. Then, from (20), we have

$$
\begin{aligned}
s_{i-1}^{k+1}-s_{i-1}^{k} & =\left(s_{i}^{k+1}-s_{i}^{k}\right)+\int_{s_{i}^{k+1}}^{s_{i}^{k+2}} \bar{F}(u) d u-\int_{s_{i}^{k}}^{s_{i}^{k+1}} \bar{F}(u) d u[\text { By (20)] } \\
& =\int_{s_{i}^{k+1}}^{s_{i}^{k+2}} \bar{F}(u) d u+\int_{s_{i}^{k}}^{s_{i}^{k+1}}(1-\bar{F}(u)) d u \\
& =\int_{s_{i}^{k+1}}^{s_{i}^{k+2}} \bar{F}(u) d u+\int_{s_{i}^{k}}^{s_{i}^{k+1}} F(u) d u \\
& \geq 0 .
\end{aligned}
$$

[Induction hypothesis]

Part (b) follows from (a) and expression (20).

For part (c), consider some valuation distribution $G$ such that $\bar{G} \geq \bar{F}$; that is, $G$ is stochastically larger than $F$. Denote $s_{i}^{k}(G)$ and $s_{i}^{k}(F)$ as the thresholds under valuation distributions $G$ and $F$, respectively. Our goal is to show that for every $i, k$,

$$
s_{i}^{k}(G) \geq s_{i}^{k}(F) .
$$

We prove (21) by induction on $i$. For $i=N, s_{i}^{k}(G)=s_{i}^{k}(F)=m(k)$, and (21) holds trivially. Inductively assume that this holds for some $i$ and for every $k$. We have

$$
\begin{aligned}
& s_{i-1}^{k}(G)-s_{i-1}^{k}(F) \\
& \left.=s_{i}^{k}(G)-s_{i}^{k}(F)+\int_{s_{i}^{k}(G)}^{s_{i}^{k+1}(G)} \bar{G}(u) d u-\int_{s_{i}^{k}(F)}^{s_{i}^{k+1}(F)} \bar{F}(u) d u \quad \text { [By }(20)\right] \\
& \geq s_{i}^{k}(G)-s_{i}^{k}(F)+\int_{s_{i}^{k}(G)}^{s_{i}^{k+1}(F)} \bar{G}(u) d u-\int_{s_{i}^{k}(F)}^{s_{i}^{k+1}(F)} \bar{F}(u) d u \quad \text { [Induction Hypothesis, } s_{i}^{k+1}(G) \geq s_{i}^{k+1}(F) \text { ] } \\
& =s_{i}^{k}(G)-s_{i}^{k}(F)-\int_{s_{i}^{k}(F)}^{s_{i}^{k}(G)} \bar{G}(u) d u+\int_{s_{i}^{k}(F)}^{s_{i}^{k+1}(F)}[\bar{G}(u)-\bar{F}(u)] d u \\
& \left.\geq s_{i}^{k}(G)-s_{i}^{k}(F)-\int_{s_{i}^{k}(F)}^{s_{i}^{k}(G)} \bar{G}(u) d u \quad \text { [By part (a) and } \bar{G} \geq \bar{F}\right] \\
& =\int_{s_{i}^{k}(F)}^{s_{i}^{k}(G)} G(u) d u \\
& \geq 0
\end{aligned}
$$

Finally, we prove (d) by induction on $i$. This holds trivially when $i=N$. Suppose that it holds for some $i$. Consider 


$$
\begin{array}{rlrl}
s_{i-1}^{k} & =s_{i}^{k}+\int_{s_{i}^{k}}^{s_{i}^{k+1}} \bar{F}(u) d u & & \text { [By (20)] } \\
& \leq s_{i}^{k}+\left(s_{i}^{k+1}-s_{i}^{k}\right) \bar{F}\left(s_{i}^{k}\right) & & \text { [By part (a), } \bar{F} \text { decreasing] } \\
& =s_{i}^{k} F\left(s_{i}^{k}\right)+s_{i}^{k+1} \bar{F}\left(s_{i}^{k}\right) & & \\
& \leq m(k+N-i) F\left(s_{i}^{k}\right)+m(k+1+N-i) \bar{F}\left(s_{i}^{k}\right) & & \text { [Induction hypothesis] } \\
& \leq m(k+N-i+1) F\left(s_{i}^{k}\right)+m(k+N-i+1) \bar{F}\left(s_{i}^{k}\right) & {[m \text { increasing }]} \\
& =m(k+N-(i-1)) . &
\end{array}
$$

Q.E.D.

Proof of Proposition 2. We prove part (a) by induction on $i$. The result holds trivially for $i=N$. Inductively assume that it holds for some $i+1$. By the induction hypothesis, $U_{i+1}$ is decreasing in $k$, and Proposition 1 (a) implies that $\mathbb{E}\left[\left(X_{i}-s_{i}(k)\right)_{+}\right]$is also decreasing in $k$. Therefore, from $(19), U_{i}(k)$ is also decreasing in $k$.

Part (b) follows immediately from (19) because $\mathbb{E}\left[\left(X_{i}-s_{i}(k)\right)_{+}\right] \geq 0$.

For (c), consider

$$
\begin{aligned}
& {\left[U_{i}(k+1)-U_{i+1}(k+1)\right]-\left[U_{i}(k)-U_{i+1}(k)\right]} \\
& =\mathbb{E}\left[\left(X_{i}-s_{i}(k+1)\right)_{+}\right]-\mathbb{E}\left[\left(X_{i}-s_{i}(k)\right)_{+}\right][\text {By }(19)] \\
& \leq 0 \quad \text { [Proposition 1(a)] }
\end{aligned}
$$

Q.E.D.

Proof of Lemma 2. Part (b) follows from using the identity that $\mathbb{E}\left[D^{1}\right]=\sum_{k=1}^{N} \mathbb{P}\left(D^{1} \geq k\right)$, and applying part (a) to substitute the expression for $\mathbb{P}\left(B_{k} \geq k\right)$. It therefore remains to prove part (a). To see this, fix $k=0, \ldots, N$, and note that by (1) and (4), we have $D^{1} \geq k \Longleftrightarrow X_{\sigma(k)} \geq m(k-1)$. Moreover, let $\left\{A_{j}\right\}_{j=0}^{N}$ be a partition where $A_{j}$ represents the event that exactly $j$ out of the $N$ random valuations exceed $m(k-1)$. Then,

$$
\begin{aligned}
\mathbb{P}\left(X_{\sigma(k)} \geq m(k-1)\right) & =\sum_{j=0}^{N} \mathbb{P}\left(\left\{X_{\sigma(k)} \geq m(k-1)\right\} \cap A_{j}\right) \\
& =\sum_{j=k}^{N} \mathbb{P}\left(\left\{X_{\sigma(k)} \geq m(k-1)\right\} \cap A_{j}\right) \\
& =\sum_{j=k}^{N} \mathbb{P}\left(B_{k}=j\right) \\
& =\mathbb{P}\left(B_{k} \geq k\right) .
\end{aligned}
$$

Q.E.D.

Proof of Lemma 3. We first prove the lower bound. For the case of $t=$ myopic, this is an immediate consequence of representation (5), and recalling that $m(\cdot)$ is increasing. For the case of $t=$ strategic, this 
is an immediate consequence of representation (9), and by recalling that $s_{i}^{k}$ is increasing in $k$ for every $i$ (Proposition 1(a)).

We next prove the upper bound by induction on $n$. For $t \in\{$ myopic,strategic $\}$, when $n=0$, because $D_{k}^{t}(0)=0$ for each $k$ (that is, nothing can be purchased if there are no periods left to purchase), (10) holds because $1+D_{k+1}^{t}(0)-D_{k}^{t}(0)=1 \geq 0$. Now suppose that (10) holds for some $n$ and every $k=0, \ldots, N-n-1$. We aim to show that (10) holds when there are $n+1$ periods remaining (this is the period when the product with random valuation $X_{N-n}$ is observed). Fix any $k=0, \ldots, N-n-2$, and define the following events:

$$
A^{\text {myopic }}:=\left\{X_{N-n} \geq m(k+1)\right\}, B^{\text {myopic }}:=\left\{m(k) \leq X_{N-n}<m(k+1)\right\}, \text { and } C^{\text {myopic }}:=\left\{X_{N-n}<m(k)\right\} .
$$

Similarly, define

$$
\begin{gathered}
A^{\text {strategic }}:=\left\{X_{N-n} \geq s_{N-n}(k+1)\right\}, B^{\text {strategic }}:=\left\{s_{N-n}(k) \leq X_{N-n}<s_{N-n}(k+1)\right\}, \\
\text { and } C^{\text {strategic }}:=\left\{X_{N-n}<s_{N-n}(k)\right\} .
\end{gathered}
$$

Note that for $t \in\{$ myopic, strategic $\},\left\{A^{t}, B^{t}, C^{t}\right\}$ form a partition of the probability space. Furthermore, for $t \in\{$ myopic, strategic $\}$,

$$
\begin{aligned}
1+D_{k+1}^{t}(n+1)-D_{k}^{t}(n+1)= & \mathbb{1}_{A^{t}}(\underbrace{1+D_{k+2}^{t}(n)-D_{k+1}^{t}(n)}_{\geq 0}) \\
& +\mathbb{1}_{B^{t}}(\underbrace{D_{k+1}^{t}(n)-D_{k+1}^{t}(n)}_{=0}) \\
& +\mathbb{1}_{C^{t}}(\underbrace{1+D_{k+1}^{t}(n)-D_{k}^{t}(n)}_{\geq 0}) \\
\geq 0, &
\end{aligned}
$$

where we have applied the induction hypothesis to establish that the first and third terms are nonnegative with probability $1 . \quad$ Q.E.D.

Proof of Lemma 4. Observe that the products purchased in periods $1,2, \ldots, j-1$ are identical in both sequences (original and interchanged); we refer to this quantity as $k$. Denote by $L$ and $\widetilde{L}$ the total number of products purchased in periods $j$ and $j+1$ from the original sequence and interchanged sequence, respectively. Recall from Lemma 3 that $D_{k}^{\text {myopic }}(n)$ denotes the number of purchases in the last $n$ remaining periods with $k$ products already purchased. Putting these together, we may write $\widetilde{D}^{\text {myopic }}-D^{\text {myopic }}=\widetilde{L}-L+D_{k+\widetilde{L}}^{\text {myopic }}(N-$ $j-1)-D_{k+L}^{\text {myopic }}(N-j-1)$. We claim that for the lemma to hold, it suffices to show that

$$
\widetilde{L}-L \in\{0,1\} \quad \text { with probability } 1 .
$$

Clearly, in the event that $\widetilde{L}=L$, then $\widetilde{D}^{\text {myopic }}=D^{\text {myopic }}$ and the result holds trivially. In the event that $\widetilde{L}-L=1$, we have $\widetilde{D}^{\text {myopic }}-D^{\text {myopic }}=1+D_{k+L+1}^{\text {myopic }}(N-j-1)-D_{k+L}^{\text {myopic }}(N-j-1) \geq 0$, where the final inequality is by Lemma 3 , and the result holds as well. 
It therefore remains to show that (22) holds. To do this, we define the following events:

$$
\begin{aligned}
& A_{11}:=\left\{X_{j} \geq m(k), X_{j+1} \geq m(k+1)\right\} \\
& A_{10}:=\left\{X_{j} \geq m(k), X_{j+1}<m(k+1)\right\} \\
& A_{00}:=\left\{X_{j}<m(k), X_{j+1}<m(k)\right\}
\end{aligned}
$$

and we will show that (22) holds on each of these sets. Namely, $A_{11}$ represents the event that both products $j$ and $j+1$ are purchased, $A_{10}$ the event that only product $j$ is purchased but not product $j+1$, and $A_{00}$ is the event that neither are purchased. Note that these are mutually exclusive events whose union has probability 1 , because $X_{j} \geq X_{j+1}$ by construction and therefore the event that product $j+1$ is purchased but product $j$ is not purchased has zero probability.

It is also useful to note the following expression for $\widetilde{L}$ :

$$
\widetilde{L}=\mathbb{1}_{\left\{X_{j+1} \geq m(k)\right\}}+\mathbb{1}_{\left\{X_{j+1} \geq m(k), X_{j} \geq m(k+1)\right\}}+\mathbb{1}_{\left\{X_{j+1}<m(k), X_{j} \geq m(k)\right\}} .
$$

If $X_{j+1} \geq m(k)$, then, in the interchanged sequence, the consumer purchases in period $j$; if so, she faces a higher threshold $m(k+1)$ in period $j+1$. This is captured in the first two terms of the expression. The last term of the expression represents the event that in the interchanged sequence, the consumer does not purchase in period $j$, which implies that she faces the same threshold $m(k)$ in period $j+1$.

To conclude the proof, first, on the set $A_{11}$, we have $L=2$. On this set, $X_{j+1} \geq m(k+1) \geq m(k)$ and $X_{j} \geq X_{j+1} \geq m(k+1)$, which implies that $\widetilde{L}=L=2$ from (23); hence, (22) holds on this set. Second, on the set $A_{10}$, we have $L=1$. On this set, $X_{j} \geq m(k)$, which implies that $\widetilde{L} \geq 1$ from (23), and therefore (22) holds on this set. Finally, on the set $A_{00}$, we have $L=0$. On this set, $X_{j+1} \leq X_{j}<m(k)$, which implies that $\widetilde{L}=L=0$ from (23) and therefore (22) holds on this set, completing the proof. Q.E.D.

Proof of Proposition 3. We prove both parts simultaneously. Given any sequence of valuations, $X_{1}, \ldots, X_{N}$, iteratively perform pairwise interchanges until the final sequence is descending (ascending) in valuations. Lemma 4 implies that each interchange results in weakly smaller (weakly greater) demand. Therefore, the final descending (ascending) sequence has minimal (maximal) demand. Q.E.D.

Proof of Theorem 1. Let $D_{\downarrow}^{\text {myopic }}$ denote the demand from a myopic consumer when she is presented with valuations in descending order, that is, $X_{\sigma(1)}, X_{\sigma(2)}, \ldots, X_{\sigma(N)}$. We will show that $D^{1}=D_{\downarrow}^{\text {myopic }}$, whence the result would follow from Proposition 3. Indeed, because $m(\cdot)$ is increasing and $X_{\sigma(\cdot)}$ is decreasing, we necessarily have $X_{\sigma(i)} \geq m(i-1)$ for every $i=1, \ldots, D_{\downarrow}^{\text {myopic }}$ and $X_{\sigma(i)}<m\left(D_{\downarrow}^{\text {myopic }}\right)$ for all $i>D_{\downarrow}^{\text {myopic }}$. Thus, using the definition of $m(\cdot)$ in $(4)$, if $D_{\downarrow}^{\text {myopic }}=0$, then we necessarily have $v(0)+X_{\sigma(1)}<p$, which implies that $D^{1}=0$ from (1). If $D_{\downarrow}^{\text {myopic }}>0$, then $D_{\downarrow}^{\text {myopic }}=\sup \left\{i: v(i-1)+X_{\sigma(i)} \geq p\right\}=D^{1}$, where the final equality is by the definition of $D^{1}$ in (1). Q.E.D. 
Proof of Theorem 2. Note that if the retailer offers a single assortment, there is nothing to prove because myopic and strategic consumers make identical decisions. In the case that the retailer offers a sequential assortment, we show that if a strategic and myopic consumer have the same realization of valuations, $\left\{X_{i}\right\}_{i=1}^{N}$ and the strategic consumer purchases some given product $i$, then a myopic consumer will also purchase product $i$. Fix some product $i=1, \ldots, N$ and suppose that each consumer (strategic, myopic) has already purchased $k$ products. The myopic consumer purchases in period $i$ if $X_{i} \geq m(k)$. The strategic consumer purchases if $X_{i} \geq s_{i}(k)$. Therefore, to prove the result, it suffices to show that $s_{i}(k) \geq m(k)$ for every $i, k$. Indeed, for every $k$, we note that $m(k)=s_{N}(k) \leq s_{i}(k)$ for any $i$ from Proposition $1(\mathrm{~b})$. Q.E.D.

Proof of Theorem 3 Note that $\mathbb{E}\left[D^{1}\right]=\mathbb{P}\left(D^{1} \geq 1\right)+\mathbb{P}\left(D^{1} \geq 2\right)$, and similarly for $D^{\text {strategic }}$. Therefore, for $D^{1}$, we have

$$
\begin{aligned}
& \mathbb{P}\left(D^{1} \geq 1\right)=\mathbb{P}\left(\max \left\{X_{1}, X_{2}\right\}>p-v(0)\right)=1-F^{2}(p-v(0))=1-F^{2}(a) \\
& \mathbb{P}\left(D^{1} \geq 2\right)=\mathbb{P}\left(\min \left\{X_{1}, X_{2}\right\}>p-v(1)\right)=\bar{F}^{2}(p-v(1))=\bar{F}^{2}(c) .
\end{aligned}
$$

For $D^{\text {strategic }}$, we first note that $b=a+\int_{a}^{c} \bar{F}(u) d u=p-v(0)-U_{2}(1)+U_{2}(0)$; we have

$$
\begin{aligned}
& \mathbb{P}\left(D^{\text {strategic }} \geq 1\right)=\mathbb{P}\left(X_{1}>b\right)+\mathbb{P}\left(X_{1} \leq b, X_{2}>a\right)=\bar{F}(b)+F(b) \bar{F}(a) \\
& \mathbb{P}\left(D^{\text {strategic }} \geq 2\right)=\mathbb{P}\left(X_{1}>b, X_{2}>c\right)=\bar{F}(b) \bar{F}(c) .
\end{aligned}
$$

Hence, $\mathbb{E}\left[D^{\text {strategic }}\right] \geq \mathbb{E}\left[D^{1}\right]$ if and only if

$$
\begin{aligned}
& \bar{F}(b)+F(b) \bar{F}(a)+\bar{F}(b) \bar{F}(c) \geq 1-F^{2}(a)+\bar{F}^{2}(c) \\
\Longleftrightarrow & \bar{F}(c)(\bar{F}(b)-\bar{F}(c)) \geq F(b)(1-\bar{F}(a))-F^{2}(a) \\
\Longleftrightarrow & \bar{F}(c)(\bar{F}(b)-\bar{F}(c)) \geq F(a)(F(b)-F(a)) .
\end{aligned}
$$

Q.E.D.

Proof of Proposition 4. We calculate $\mathbb{E}\left[D^{1}\right]$ similarly to Theorem 3 , by using $\mathbb{E}\left[D^{1}\right]=\sum_{k=1}^{3} \mathbb{P}\left(D^{1} \geq k\right)$ and evaluating each term of the sum directly. For $\mathbb{E}\left[D^{\text {strategic }}\right]$, we first calculate the thresholds by applying (20) repeatedly, and subsequently calculate $\mathbb{E}\left[D^{\text {strategic }}\right]$ by recursively evaluating the expected number of items purchased in each period, starting from the last period. We then evaluate $g$ as $\mathbb{E}\left[D^{\text {strategic }}\right]-\mathbb{E}\left[D^{1}\right]=$ $K g(x, y, z)$, where $K$ is a scaling constant. This gives us the following expression for $g$ : 


$$
\begin{aligned}
g(x, y, z):= & x^{7}-x^{6} y+x^{6}-3 x^{5} y^{2}+6 x^{5} y+2 x^{4} y^{3}+x^{4} y^{2} z-10 x^{4} y^{2}+x^{4} y z^{2}-2 x^{4} y z+8 x^{4} y-x^{4} z^{3} \\
& +3 x^{4} z^{2}-4 x^{4} z+2 x^{4}+2 x^{3} y^{4}-12 x^{3} y^{3}+2 x^{3} y^{2} z^{2}-4 x^{3} y^{2} z+16 x^{3} y^{2}-x^{3} z^{4} \\
& +4 x^{3} z^{3}-8 x^{3} z^{2}+8 x^{3} z-16 x^{3}-2 x^{2} y^{4} z+12 x^{2} y^{4}-4 x^{2} y^{3} z^{2}+12 x^{2} y^{3} z-36 x^{2} y^{3} \\
& +2 x^{2} y^{2} z^{3}-4 x^{2} y^{2} z+20 x^{2} y^{2}+x^{2} y z^{4}-8 x^{2} y z^{3}+20 x^{2} y z^{2}-24 x^{2} y z+8 x^{2} y-x^{2} z^{4}+ \\
& +4 x^{2} z^{3}-8 x^{2} z^{2}+8 x^{2} z+4 x y^{5}-2 x y^{4} z^{2}+4 x y^{4} z-16 x y^{4}+4 x y^{3} z^{2}-8 x y^{3} z+16 x y^{3}+x y^{2} z^{4} \\
& -4 x y^{2} z^{3}+8 x y^{2} z^{2}-8 x y^{2} z-2 x y z^{4}+8 x y z^{3}-16 x y z^{2}+16 x y z-4 y^{5} z+16 y^{5}+2 y^{4} z^{3}-16 y^{4} z^{2} \\
& +32 y^{4} z-48 y^{4}+2 y^{3} z^{4}-4 y^{3} z^{3}+12 y^{3} z^{2}-16 y^{3} z+64 y^{3}-3 y^{2} z^{5}+18 y^{2} z^{4}-52 y^{2} z^{3}+84 y^{2} z^{2} \\
& -80 y^{2} z-24 y^{2}-y z^{6}+6 y z^{5}-20 y z^{4}+40 y z^{3}-56 y z^{2}+48 y z+z^{7}-7 z^{6}+24 z^{5} \\
& -50 z^{4}+56 z^{3}-24 z^{2},
\end{aligned}
$$

which can be seen to be a 7 th degree polynomial in the three variables $(x, y, z)$. Q.E.D.

Proof of Theorem 4. Throughout this proof, for notational brevity, we write $s_{i}^{k}:=s_{i}(k)$. Recall from Lemma 3 that $D_{k}^{\text {strategic }}(n)$ represents the number of products purchased by the strategic consumer in the last $n$ assortments of the season, if she has already purchased $k$ products. Note that $D^{\text {strategic }}=D_{0}^{\text {strategic }}(N)$, and that if she has $n$ assortments left to view, then in the dynamic programming formulation (6), she is in period $N-n+1$.

To prove (a), we begin with a definition and a preliminary result. For $n=0, \ldots, N$ and $k=0, \ldots, N-n-1$, define

$$
\delta_{n}^{k}:=1+\mathbb{E}\left[D_{k+1}^{\text {strategic }}(n)\right]-\mathbb{E}\left[D_{k}^{\text {strategic }}(n)\right] .
$$

Note that Lemma 3 implies that $\delta_{n}^{k} \geq 0$ for each $n, k$.

We can rearrange the definition of $\delta_{n}^{k}$, and by conditioning on whether a purchase was made or not in period $N-n$, we get

$$
\mathbb{E}\left[D_{k}^{\text {strategic }}(n+1)\right]=\mathbb{E}\left[D_{k}^{\text {strategic }}(n)\right]+\bar{F}\left(s_{N-n}^{k}\right) \delta_{n}^{k}
$$

We claim that

$$
\delta_{n+1}^{k} \geq \bar{F}\left(s_{N-n}^{k+1}\right) \delta_{n}^{k+1}
$$

To see this, consider

$$
\begin{array}{rlr}
\delta_{n+1}^{k} & =1+\mathbb{E}\left[D_{k+1}^{\text {strategic }}(n+1)\right]-\mathbb{E}\left[D_{k}^{\text {strategic }}(n+1)\right] & {[\text { By (24)] }} \\
& =1+\mathbb{E}\left[D_{k+1}^{\text {strategic }}(n)\right]+\bar{F}\left(s_{N-n}^{k+1}\right) \delta_{n}^{k+1}-\mathbb{E}\left[D_{k}^{\text {strategic }}(n)\right]-\bar{F}\left(s_{N-n}^{k}\right) \delta_{n}^{k} & {[\text { By }(25)]} \\
& =\delta_{n}^{k}+\bar{F}\left(s_{N-n}^{k+1}\right) \delta_{n}^{k+1}-\bar{F}\left(s_{N-n}^{k}\right) \delta_{n}^{k} & \\
& =\bar{F}\left(s_{N-n}^{k+1}\right) \delta_{n}^{k+1}+F\left(s_{N-n}^{k}\right) \delta_{n}^{k} & \\
& \geq \bar{F}\left(s_{N-n}^{k+1}\right) \delta_{n}^{k+1}, & {\left[\delta_{n}^{k} \geq 0\right. \text { by }}
\end{array}
$$

$\left[\delta_{n}^{k} \geq 0\right.$ by Lemma 3]

thus establishing (26). 
Recalling that $D^{\text {strategic }}=D_{0}^{\text {strategic }}(N)$, we apply (25) for the case $k=0, n=N-1$ to get

$$
\mathbb{E}\left[D_{0}^{\text {strategic }}(N)\right]=\mathbb{E}\left[D_{0}^{\text {strategic }}(N-1)\right]+\bar{F}\left(s_{1}^{0}\right) \delta_{N-1}^{0} .
$$

Next, we claim that under the assumption that $X_{i} \geq m(N-2)$ a.s., we necessarily have $D_{0}^{\text {strategic }}(N-1)=$ $N-1$ with probability 1 . This is because we can equivalently interpret $D_{0}^{\text {strategic }}(N-1)$ as the demand from a strategic consumer who only views $N-1$ assortments. Hence, we can use representation (9) for $D_{0}^{\text {strategic }}(N-1)$, which yields

$$
D_{0}^{\text {strategic }}(N-1)=\sum_{i=1}^{N-1} \mathbb{1}_{\left\{X_{i} \geq s_{i}\left(Z_{i-1}\right)\right\}},
$$

where $Z_{j} \leq j$ for each $j=0, \ldots, N-1$. Consider

$$
\begin{aligned}
s_{i}\left(Z_{i-1}\right) & \leq m\left(Z_{i-1}+N-1-i\right) \\
& \leq m(\text { Proposition } 1(\mathrm{~d}) \text { for } N-1] \\
& {\left[Z_{i-1} \leq i-1, m(\cdot)\right. \text { increasing] }}
\end{aligned}
$$

Therefore, $D_{0}^{\text {strategic }}(N-1)=N-1$ with probability 1 .

For the single assortment, apply (2) and the hypothesis that $X_{i} \geq m(N-2)$ with probability 1 to get

$$
\mathbb{E}\left[D^{1}\right]=\mathbb{E}\left[\sum_{i=1}^{N} \mathbb{1}_{\left\{X_{\sigma(i)} \geq m(i-1)\right\}}\right]=(N-1)+\mathbb{P}\left(X_{\sigma(N)} \geq m(N-1)\right)=(N-1)+\bar{F}^{N}(m(N-1)) .
$$

Comparing the expressions for $\mathbb{E}\left[D^{\text {strategic }}\right]$ and $\mathbb{E}\left[D^{1}\right]$, and noting that from Proposition $1(\mathrm{~d})$, we have $s_{1}(0) \leq m(N-1)$, and since $\bar{F}$ is decreasing, $\bar{F}(m(N-1)) \leq \bar{F}\left(s_{1}(0)\right)$. Therefore, to complete the proof, it suffices to show that

$$
\delta_{N-1}^{0} \geq \bar{F}^{N-1}(m(N-1)) .
$$

We will do this by proving that for every $n=0, \ldots, N-1$ and $k=N-1-n$, we have

$$
\delta_{n}^{k} \geq \bar{F}^{n}(m(N-1)),
$$

of which (27) is a special case.

We prove (28) by induction on $n$. The case of $n=0$ holds trivially. Suppose that (28) holds for some $n$, and we aim to show it for $n+1$. Then,

$$
\begin{array}{rlrl}
\delta_{n+1}^{k-1} & \geq \bar{F}\left(s_{N-n}^{k}\right) \delta_{n}^{k} & & {[\text { By (26)] }} \\
& \geq \bar{F}\left(s_{N-n}^{k}\right) \bar{F}^{n}(m(N-1)) & & \text { [Induction hypothesis] } \\
& \geq \bar{F}(m(k+n)) \bar{F}^{n}(m(N-1)) & \text { [Proposition 1(d) and } \bar{F} \text { decreasing] } \\
& =\bar{F}^{n+1}(m(N-1)) & & {[k=N-1-n \text { by assumption] }}
\end{array}
$$

Therefore, (27) holds, and hence, $\mathbb{E}\left[D^{\text {strategic }}\right] \geq \mathbb{E}\left[D^{1}\right]$.

To prove (b), we first note that in this setting,

$$
\mathbb{E}\left[D^{1}\right]=1-F^{N}(m(0)) .
$$


Furthermore, for any $i \geq 1, k \geq 1$ we have $s_{i}^{k} \geq s_{N}^{k}=m(k) \geq m(1)$, where the first inequality is by Proposition $1(\mathrm{~b})$, and the second because $m(\cdot)$ is increasing, which implies that $\bar{F}\left(s_{i}^{k}\right) \leq \bar{F}(m(1))$. Because we have assumed that $X_{i} \leq m(1)$ a.s., this implies that for $k \geq 1$, we necessarily have $\bar{F}\left(s_{i}^{k}\right)=0$ for any $i \geq 1$. In particular, using (25), this implies that for $k \geq 1$, and any $n=0, \ldots, N-1$, we have

$$
\mathbb{E}\left[D_{k}^{\text {strategic }}(n+1)\right]=\mathbb{E}\left[D_{k}^{\text {strategic }}(n)\right]=\ldots=\mathbb{E}\left[D_{k}^{\text {strategic }}(0)\right]=0
$$

Now we use the definition of $\delta_{n}^{k}$ from (24) to expand (25) for the case of $k=0$ to get

$$
\mathbb{E}\left[D_{0}^{\text {strategic }}(n+1)\right]=\bar{F}\left(s_{N-n}^{0}\right)+F\left(s_{N-n}^{0}\right) \mathbb{E}\left[D_{0}^{\text {strategic }}(n)\right]
$$

Defining $Q_{n}:=1-\mathbb{E}\left[D_{0}^{\text {strategic }}(n)\right]$, the above may be written as $Q_{n+1}=F\left(s_{N-n}^{0}\right) Q_{n}$, for $n=0, \ldots, N-1$. Since $Q_{0}:=1$, we have

$$
Q_{N}=\prod_{n=0}^{N-1} F\left(s_{N-n}^{0}\right) \geq \prod_{n=0}^{N-1} F(m(0))=F^{N}(m(0)),
$$

where the inequality is by Proposition $1(\mathrm{~b})$ and because $F$ is increasing. Therefore, we conclude that

$$
\mathbb{E}\left[D^{\text {strategic }}\right]=\mathbb{E}\left[D_{0}^{\text {strategic }}(N)\right]=1-Q_{N} \leq 1-F^{N}(m(0))=\mathbb{E}\left[D^{1}\right]
$$

Q.E.D.

Proof of Theorem 5. For this proof, we denote $m(\infty):=\lim _{k \rightarrow \infty} m(k)$, and note that $m(\infty)$ is finite because $v$ is assumed to be decreasing and bounded from below. Furthermore, note that although we do not denote this explicitly, $D^{t}$ for each $t \in\{1$, myopic, strategic $\}$ all depend on $N$.

First, we prove part (a) for $t=$ myopic. Construct the random variable $B_{N}$ as

$$
B_{N}:=\sum_{i=1}^{N} \mathbb{1}_{\left\{X_{i} \geq m(\infty)\right\}}
$$

Furthermore, recalling the definition of the process $\left\{Y_{i}\right\}_{i=0}^{N}$ from the construction (5), for an arbitrary $K$, construct the random variable $B_{N}^{K}$ as

$$
B_{N}^{K}:=\sum_{i=1}^{N} \mathbb{1}_{\left\{X_{i} \geq m\left(\min \left\{Y_{i-1}, K\right\}\right)\right\}} .
$$

By coupling, since $m\left(\min \left\{Y_{i}, K\right\}\right) \leq m\left(Y_{i}\right) \leq m(\infty)$, we have

$$
B_{N} \leq D^{\text {myopic }} \leq B_{N}^{K}
$$

Define the (integer-valued) random variable $L_{N}^{K}$ as $L_{N}^{K}:=\sup \left\{i \in\{1, \ldots, N+1\}: Y_{i-1}<K\right\}$, so that we can decompose $B_{N}^{K}$ as

$$
B_{N}^{K}=\sum_{i=1}^{L_{N}^{K}} \mathbb{1}_{\left\{X_{i} \geq m\left(Y_{i-1}\right)\right\}}+\sum_{i=L_{N}^{K}+1}^{N} \mathbb{1}_{\left\{X_{i} \geq m(K)\right\}}
$$


By the Strong Law of Large Numbers, we have $B_{N} / N \stackrel{\text { a.s. }}{\rightarrow} \delta$. From the lower bound of (29), and recalling from (5) that $D^{\text {myopic }}=Y_{N}$, we necessarily have $\lim _{i \rightarrow \infty} Y_{i}=\infty$ with probability 1 , which implies that $\lim _{N \rightarrow \infty} L_{N}^{K}$ is bounded almost surely. Hence,

$$
\begin{array}{rlr}
\lim _{N \rightarrow \infty} \frac{B_{N}^{K}}{N} & =\lim _{N \rightarrow \infty} \frac{1}{N} \sum_{i=1}^{L_{N}^{K}} \mathbb{1}_{\left\{x_{i} \geq m\left(Y_{i-1}\right)\right\}}+\lim _{N \rightarrow \infty} \frac{1}{N} \sum_{i=L_{N}^{K}+1}^{N} \mathbb{1}_{\left\{x_{i} \geq m(K)\right\}} \\
& =\lim _{N \rightarrow \infty} \frac{1}{N} \sum_{i=L_{N}^{K}+1}^{N} \mathbb{1}_{\left\{X_{i} \geq m(K)\right\}} \quad\left[\lim _{N \rightarrow \infty} L_{N}^{K}\right. \text { is a.s. bounded] } \\
& \leq \lim _{N \rightarrow \infty} \frac{1}{N} \sum_{i=0}^{N-1} \mathbb{1}_{\left\{x_{i+1} \geq m(K)\right\}} \\
& =\mathbb{P}\left(X_{i} \geq m(K)\right)
\end{array}
$$

where the final equality is again by the Strong Law of Large Numbers. Therefore, we have shown that with probability 1 ,

$$
\delta \leq \lim _{N \rightarrow \infty} D^{\text {myopic }} / N \leq \mathbb{P}\left(X_{i} \geq m(K)\right) .
$$

We complete the proof by sending $K$ to $+\infty$ on the RHS.

To prove (a) for $t=$ strategic, we simply note that Proposition 1 (d) implies that $s_{i}(k) \leq m(\infty)$ for every $i, k$. Hence, from the expression for $D^{\text {strategic }}$ in (9), through coupling, we again have $B_{N} \leq D^{\text {strategic }}$. Furthermore, recall from Theorem 2 that $D^{\text {strategic }} \leq D^{\text {myopic }}$. Hence, the result follows from the myopic case by a sandwich argument.

To prove (a) for $t=1$, we note that $B_{N}$ can be equivalently written as $B_{N}=\sum_{i=1}^{N} \mathbb{1}_{\left\{X_{\sigma(i)} \geq m(\infty)\right\}}$, and we therefore have $B_{N} \leq D^{1}$ by coupling and $D^{1} \leq D^{\text {myopic }}$ by Theorem 1 . Hence, the result follows from the myopic case by a sandwich argument.

To prove (b), consider $\widetilde{v}$ that is constructed as $\widetilde{v}(k):=\max \left\{v(k), v\left(N_{0}-1\right)\right\}$ for all $k$, and for each $t \in$ $\{1$, myopic, strategic $\}$ let $\widetilde{D}^{t}$ represent the corresponding demand when $\widetilde{v}$ is used instead of $v$. Since $\widetilde{v} \geq v$, we necessarily have $D^{t} \leq \widetilde{D}^{t}$ by coupling, and in particular, $\mathbb{1}_{\left\{\widetilde{D}^{t} \leq N_{0}\right\}} D^{t}=\mathbb{1}_{\left\{\widetilde{D}^{t} \leq N_{0}\right\}} \widetilde{D}^{t}$. Therefore, noting that $D^{t} \leq N_{0}$ almost surely, we may write

$$
D^{t}=\min \left\{\widetilde{D}^{t}, N_{0}\right\}
$$

Notice that by construction of $\widetilde{v}$, we necessarily have $\lim _{N \rightarrow \infty} \mathbb{P}\left(X_{i} \geq p-\widetilde{v}(N-1)\right)=\lim _{N \rightarrow \infty} \mathbb{P}\left(X_{i} \geq p-v\left(N_{0}-1\right)\right)>$ 0 , where the final inequality is by construction of $N_{0}$. This implies that we can apply part (a) for $\widetilde{D}^{t}$, which in turn implies that with probability $1, \lim _{N \rightarrow \infty} \widetilde{D}^{t}=+\infty$. Therefore, using this with (30) implies that with probability $1, \lim _{N \rightarrow \infty} D^{t}=N_{0}$. Q.E.D. 


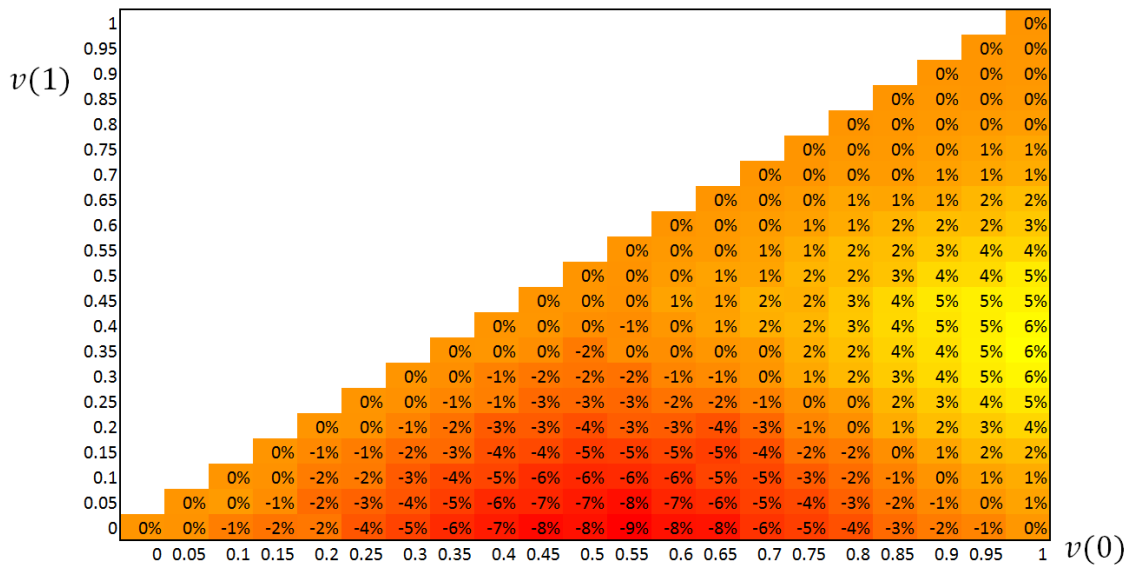

Figure 5 Value of concealment as a percentage of expected revenue from the single assortment strategy: $p=1$ and $X \sim \operatorname{Normal}\left(\mu=0.5, \sigma^{2}=\frac{1}{12}\right)$

APPENDIX B: Numerical Results for $N=2, X_{i} \sim \operatorname{Normal}\left(\mu=0.5, \sigma^{2}=\frac{1}{12}\right)$

Here we present numerical results when $X_{i} \sim \operatorname{Normal}\left(\mu=0.5, \sigma^{2}=\frac{1}{12}\right)$; note that the mean and variance of this distribution are identical to the mean and variance of the $\operatorname{Uniform}(0,1)$ distribution used in Figure 3 . Figure 5 shows a heat map of the value of concealment, expressed as a percentage of the retailer's expected revenue from following the single assortment strategy, for the case where $p=1, X \sim \operatorname{Normal}\left(\mu=0.5, \sigma^{2}=\right.$ $\left.\frac{1}{12}\right)$, and for different values of $v(0) \in[0,1], v(1) \in[0, v(0)]$. These numerical results are averages of 10,000 simulation runs per set of parameters.

As in Figure 3, the yellow region of the heat map in Figure 5 represents values of $v(0)$ and $v(1)$ where the value of concealment is positive, and the red region of the heat map represents values of $v(0)$ and $v(1)$ where the value of concealment is negative. The line $v(0)+v(1)=1$ separates the yellow and red regions as it did in Figure 3. Comparing Figures 3 and 5 suggests that our intuition extends beyond the case where $X_{i} \sim \operatorname{Uniform}(\alpha, \beta)$ to the case where $X_{i} \sim \operatorname{Normal}\left(\mu, \sigma^{2}\right)$, and we conjecture that these insights are broadly applicable to many sets of parameters over the consumer's valuations. 